\title{
Dissimilarity Cumulation Theory in Smoothly Connected Spaces
}

\author{
Ehtibar N. Dzhafarov* \\ Purdue University
}

\begin{abstract}
This is the third paper in the series introducing the Dissimilarity Cumulation theory and its main psychological application, Universal Fechnerian Scaling. The previously developed dissimilarity-based theory of path length is used to construct the notion of a smooth path, defined by the property that the ratio of the dissimilarity between its points to the length of the subtended fragment of the path tends to unity as the points gets closer to each other. We consider a class of stimulus spaces in which for every path there is a series of piecewise smooth paths converging to it pointwise and in length; and a subclass of such spaces where any two sufficiently close points can be connected by a smooth "geodesic in the small." These notions are used to construct a broadly understood Finslerian geometry of stimulus spaces representable by regions of Euclidean $n$-spaces. With an additional assumption of comeasurability in the small between the canonical psychometric increments of the first and second kind, this establishes a link between Universal Fechnerian Scaling and Multidimensional Fechnerian Scaling in Euclidean $n$-spaces. The latter was a starting point for our theoretical program generalizing Fechner's idea that sensation magnitudes can be computed by integration of a local discriminability measure.

KEYWORDS: arc length, convexity, discrimination probability, dissimilarity, Fechnerian Scaling, geodesic, Finsler geometry, indicatrix, oriented distance, perceptual discrimination, Regular Minimality, samedifferent judgements, smooth path, stimulus space.
\end{abstract}

\section{Introduction}

This is the third in a series of papers dealing with the mathematical theory of Dissimilarity Cumulation (DC) and its application to pairwise discrimination probabilities, termed Universal Fechnerian Scaling (UFS). In the first paper of the series (Dzhafarov \& Colonius, 2007) we introduced the notion of dissimilarity function and showed how it imposes a topology, uniformity, and metric on stimulus spaces. Relevant aspects of this paper are recapitulated in Section 2.2. Some of them are also mentioned, briefly and informally, in Section 1.1. In the second paper (Dzhafarov, 2007) the general DC theory was used to introduce the notion of the

\footnotetext{
${ }^{*}$ Department of Psychological Sciences, Purdue University, 703 Third Street, West Lafayette, IN 47907-2081, USA. E-mail address: ehtibar@purdue.edu (E.N. Dzhafarov).
} 
path length and to specialize the theory to stimulus spaces in which points can be connected by continuous paths. Elements of this theory are informally outlined in Section 1.2 and more rigorously recapitulated in Section 2.3 .

The present paper is an attempt to further specialize the DC theory to spaces with appropriately defined smooth paths, and to arrive through a series of intermediate constructions to a Finslerian theory of subjective (Fechnerian) distances which was the starting point for our theoretical program of Generalized Fechnerian Scaling (Dzhafarov, 2002a-d; Dzhafarov \& Colonius, 1999, 2001).

The recapitulation of the two previous papers given below is by necessity schematic and incomplete. It is merely a list of definitions and relevant results, with justifications, proofs, and detailed explanations left out. Although this list makes the present paper formally self-sufficient, the reader should consult the previous papers to understand the general context and motivations for our approach (the specific motivation for the specialization of the DC and UFS theories to smoothly connected spaces is given in Section 1.3).

All special terms mentioned in the rest of this Introduction are rigorously defined in the subsequent sections. The two abbreviations, DC and UFS, are used throughout the paper.

\subsection{Basics of Dissimilarity Cumulation (Informal Account)}

The main idea of Fechner's original theory (Fechner, 1860, 1877, 1887) can be interpreted as shown in Fig. 1 (Dzhafarov, 2001; Dzhafarov \& Colonius, 1999). If stimuli are represented by an interval of real numbers, as Fechner always assumed, the subjective distance from a stimulus $a$ to stimulus $b$ is computed as follows: every stimulus $x$ is characterized by its dissimilarity from its "immediate" neighbors on the right (e.g., the derivative with respect to $y$ of the probability with which $y$ is judged to be greater than $x$, taken at $y=x$ ), and this dissimilarity value is cumulated (integrated) as $x$ moves from $a$ to be $b$ through all intermediate stimuli.

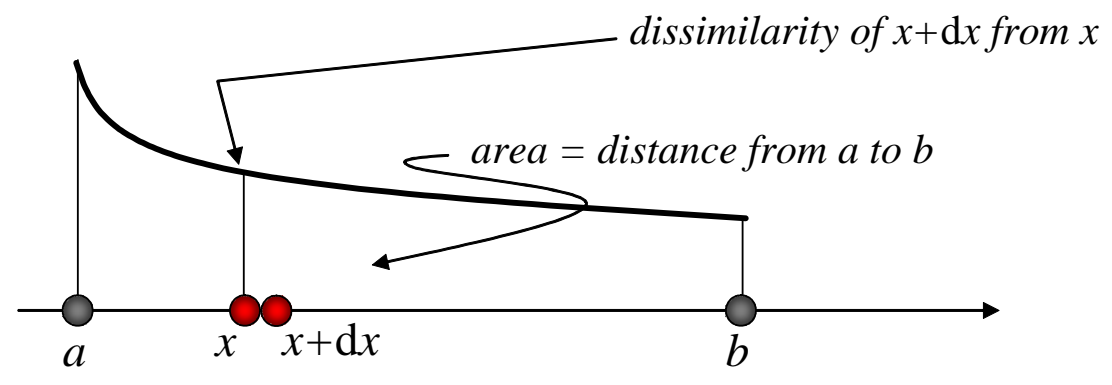

Figure 1. A possible interpretation of Fechner's main idea. To compute the subjective (Fechnerian) distance from $a$ to $b$ on a stimulus continuum, one cumulates (here, integrates) the dissimilarity of $x$ from its "infinitesimally close" neighbors on the right as $x$ varies from $a$ to $b$.

The DC theory proposed in Dzhafarov and Colonius (2007) can be viewed as a sweeping generalization of this idea of cumulating dissimilarities as one moves in a stimulus space from one stimulus to another through 


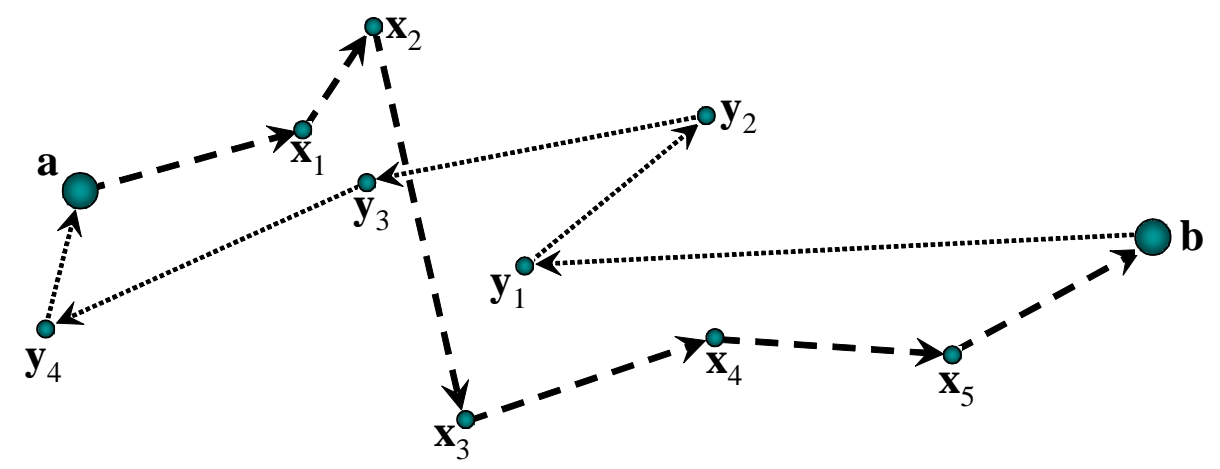

Figure 2. An illustration for dissimilarity cumulation and the computation of distances. Trying all possible chains leading from $\mathbf{a}$ to $\mathbf{b}$ in the stimulus space (shown is a chain $\mathbf{a x}_{1} \mathbf{x}_{2} \mathbf{x}_{3} \mathbf{x}_{4} \mathbf{x}_{5} \mathbf{b}$, dashed line) and finding the infimum of the corresponding cumulated dissimilarities (the cumulated dissimilarity for the chain shown is $D \mathbf{a x}_{1}+D \mathbf{x}_{1} \mathbf{x}_{2}+\ldots+D \mathbf{x}_{5} \mathbf{b}$ ) one computes the oriented distance $G \mathbf{a b}$ from $\mathbf{a}$ to $\mathbf{b}$. The cumulated dissimilarity for chains leading from $\mathbf{b}$ to $\mathbf{a}$ ( $\operatorname{shown}$ is a chain $\mathbf{b y}_{1} \mathbf{y}_{2} \mathbf{y}_{3} \mathbf{y}_{4} \mathbf{a}$, point line) and the oriented distance $G \mathbf{b a}$ are determined analogously. The overall, symmetrical distance $G^{*} \mathbf{a b}$ between $\mathbf{a}$ and $\mathbf{b}$ can be thought of as $G \mathbf{a b}+G \mathbf{b a}$, or as the infimum of the dissimilarities cumulated along all closed chains of stimuli containing $\mathbf{a}$ and $\mathbf{b}$.

intermediate stimuli. Given an arbitrary set of stimuli of a certain kind (say, colors or human faces), any two stimuli $\mathbf{a}, \mathbf{b}$ in it are characterized by a (generally asymmetric) degree of dissimilarity $D \mathbf{a b}{ }^{1}{ }^{1}$ In computing the Fechnerian distance from $\mathbf{a}$ to $\mathbf{b}$, however, one has to consider all possible ways $\mathbf{a x}_{1} \ldots \mathbf{x}_{k} \mathbf{b}$ of getting from $\mathbf{a}$ to $\mathbf{b}$ (see Fig. 2) and compare all the corresponding values of cumulated dissimilarity

$$
D \mathbf{a x}_{1} \ldots \mathbf{x}_{k} \mathbf{b}=D \mathbf{a x}_{1}+D \mathbf{x}_{1} \mathbf{x}_{2}+\ldots+D \mathbf{x}_{k} \mathbf{b}
$$

The Fechnerian distance $G \mathbf{a b}$ from $\mathbf{a}$ to $\mathbf{b}$ then is posited to be the smallest value of $D \mathbf{a x}_{1} \ldots \mathbf{x}_{k} \mathbf{b}$ achievable in this way (more precisely, the greatest lower boundary for $D \mathbf{a x}_{1} \ldots \mathbf{x}_{k} \mathbf{b}$ over all possible routes $\mathbf{x}_{1} \ldots \mathbf{x}_{k}$ ). If this infimum equals $D \mathbf{a b}$, then the dissimilarity $D$ and distance $G$ coincide. In other cases $D \mathbf{a x}_{1} \ldots \mathbf{x}_{k} \mathbf{b}$ for some nonempty chain of stimuli $\mathbf{x}_{1} \ldots \mathbf{x}_{k}$ may be smaller than Dab: then the Fechnerian distance $G \mathbf{a b}$ is smaller than $D \mathbf{a b}$. It is possible in some stimulus spaces that the infimum of $D \mathbf{a x}_{1} \ldots \mathbf{x}_{k} \mathbf{b}$ can only be approached as $k \rightarrow \infty$ and the cumulated dissimilarities $D \mathbf{a x}_{1}, D \mathbf{x}_{1} \mathbf{x}_{2}, \ldots, D \mathbf{x}_{k} \mathbf{b}$ all tend to zero. In such cases the finite chains of stimuli connecting $\mathbf{a}$ to $\mathbf{b}$ may be replaced by continuous paths, as discussed in Sections 1.2 and 2.3 .

The Fechnerian distance Gab is an oriented distance, meaning that it has all defining properties of a metric except for symmetry. The overall (symmetric) Fechnerian distance between $\mathbf{a}$ and $\mathbf{b}$ is computed as

$$
G^{*} \mathbf{a b}=G \mathbf{a b}+G \mathbf{b a} .
$$

The reason for this additive symmetrization scheme is given in Section 2.2. If the dissimilarity $D$ is itself a symmetric distance, then $G \mathbf{a b}=D \mathbf{a b}$ and $G^{*} \mathbf{a b}=2 D \mathbf{a b}$.

\footnotetext{
${ }^{1}$ Although the notation we use should be clear from the context, the reader may consult Section 2.1 where the notation conventions are stated explicitly.
} 
The notion of a dissimilarity Dab therefore is more general than that of a distance, and it is used as a building block for computing distances among stimuli. Essentially, dissimilarity Dab is a nonnegative uniformly continuous function vanishing only at $\mathbf{a}=\mathbf{b}$ and having the following property: consider a sequence of stimulus chains

$$
\mathbf{X}_{1}=\mathbf{x}_{1}^{1} \ldots \mathbf{x}_{k_{1}}^{1}, \quad \mathbf{X}_{2}=\mathbf{x}_{1}^{2} \ldots \mathbf{x}_{k_{2}}^{2}, \quad \ldots, \quad \mathbf{X}_{n}=\mathbf{x}_{1}^{n} \ldots \mathbf{x}_{k_{n}}^{n}, \quad \ldots
$$

with elements and their numbers varying from one chain to another; denote the cumulated dissimilarity along the chain $\mathbf{X}_{n}$ (also referred to as the D-length of the chain $\mathbf{X}_{n}$ ) by

$$
D \mathbf{X}_{n}=D \mathbf{x}_{1}^{n} \mathbf{x}_{2}^{n}+D \mathbf{x}_{2}^{n} \mathbf{x}_{3}^{n}+\ldots+D \mathbf{x}_{k_{n}-1}^{n} \mathbf{x}_{k_{n}}^{n}
$$

the property in question is that $D \mathbf{X}_{n}$ can gradually vanish (converge to zero as $n \rightarrow \infty$ ) only if the dissimilarity $D \mathbf{x}_{1}^{n} \mathbf{x}_{k_{n}}^{n}$ of its terminal element from its initial element gradually vanishes too.

When applied to discrimination probabilities $\psi \mathbf{a b}$ (the probability with which $\mathbf{a}$ and $\mathbf{b}$ are judged to be different), the canonical psychometric increments

$$
\begin{aligned}
& \Psi^{(1)} \mathbf{a b}=\psi \mathbf{a b}-\psi \mathbf{a} \mathbf{a} \\
& \Psi^{(2)} \mathbf{a b}=\psi \mathbf{b} \mathbf{a}-\psi \mathbf{a} \mathbf{a}
\end{aligned}
$$

are posited to be, both of them, dissimilarity functions. ${ }^{2}$ The term "canonical" refers to an appropriately chosen labeling of the stimuli, under which

$$
\psi \mathbf{a a}<\min \{\psi \mathbf{a b}, \psi \mathbf{b a}\}
$$

for any distinct a, b. The law of Regular Minimality (Dzhafarov, 2002d, 2006; Dzhafarov \& Colonius, 2006a) which we posit as a cornerstone principle of UFS guarantees that such a labeling (a canonical representation of stimuli) is possible. ${ }^{3}$ The overall (symmetric) metric $G^{*} \mathbf{a b}$ computed from either of the two functions $\Psi^{(1)}$ and $\Psi^{(2)}$ (the value of $G^{*}$ ab being the same in either case) is interpreted as the subjective distance between $\mathbf{a}$ and $\mathbf{b}$.

\subsection{Path Length in General (Informal Account)}

In the second paper of the series (Dzhafarov, 2007) the DC theory was applied to spaces in which points can be connected by continuous paths (with respect to the topology and uniformity imposed on stimuli by the dissimilarity function, as described in Section 2.2). The D-length of a path is defined as the lower limit

\footnotetext{
${ }^{2}$ We call $\Psi^{(1)}$ and $\Psi^{(2)}$ the (canonical) psychometric increments of, resp., the first kind and the second kind. Anything computed from $\Psi^{(1)}$ (or $\Psi^{(2)}$ ) then acquires the designation "of the first kind" (resp., "of the second kind"). One of the important features of UFS is that the overall (symmetrical) distance $G^{*} \mathbf{a b}$ is the same whether it is computed from $\Psi^{(1)}$ or $\Psi^{(2)}$.

${ }^{3}$ Although we do not emphasize this topic in this paper, the physical identities of the first and second $\mathbf{a}$ in (a, a) need not be the same: the identity of a stimulus is encoded by its label and its observation area, the latter corresponding to its position in an ordered pair of stimuli (Dzhafarov, 2002d; Dzhafarov \& Colonius, 2006a, 2007).
} 
of the $D$-lengths of finite chains which converge to the path in some well-defined sense (Fig. 3 ). If $D$ itself is a metric, the theory reduces to the well-known metric-based theory of path length (Blumenthal, 1953; Blumenthal \& Menger, 1970; Busemann, 2005). In this special case the length of a path is simply the limit length of the chains inscribed in the path (i.e., the chains whose elements belong to the path's image). In general, however, the chains converging to a path need not be inscribed (as one can see in Fig. 3). With this important difference, it is shown in Dzhafarov (2007) that all the principal results of a metric-based theory of path length also hold in the general, dissimilarity-based theory. This means that these results are not critically based on the triangle inequality and symmetry, the two defining properties of a metric which a dissimilarity function need not possess.

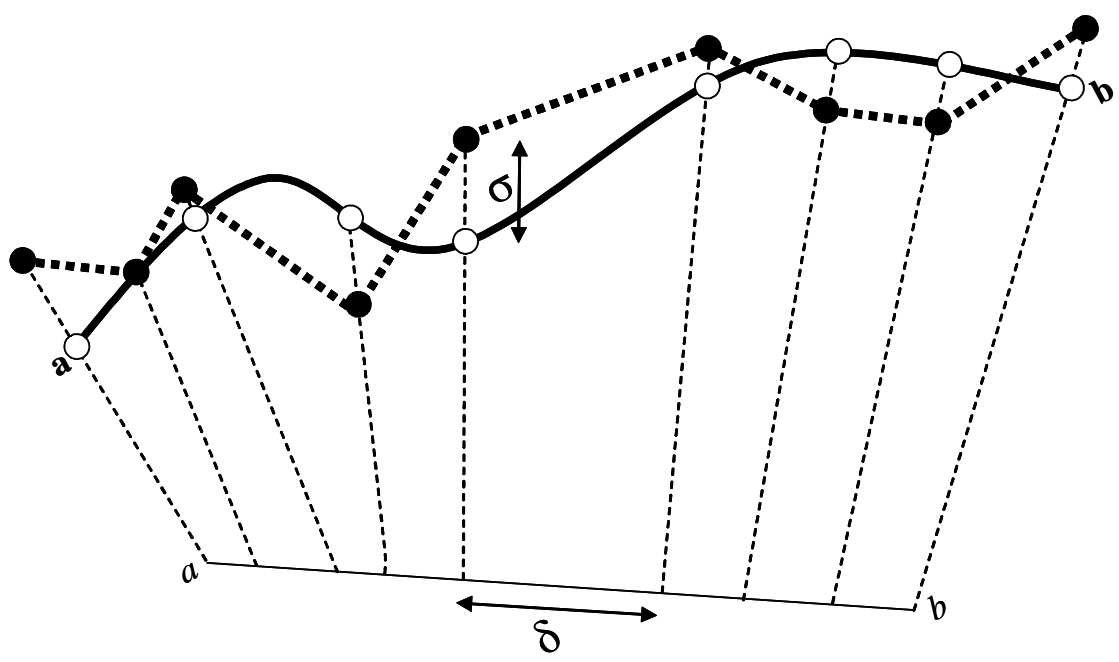

Figure 3. An illustration for the convergence of chains (more precisely, chains-on-nets, as defined in Section 2.3) to a path. The path is shown as a continuous mapping of an interval $[a, b]$ to a stimulus space, with the image indicated by the thick curve. The chain is indicated by closed circles assigned to points in $[a, b]$ and connected by thick point lines. The convergence means that $\sigma \rightarrow 0$ (where $\sigma$ is the largest dissimilarity between an element of the chain and the corresponding point on the path) and $\delta \rightarrow 0$ (where $\delta$ is the largest interval in the net of points to which the elements of the chain are assigned). The $D$-length of the path is the limit inferior of the $D$-lengths of all such converging chains.

As mentioned earlier, for some stimulus spaces endowed with dissimilarity functions the finite chains of stimuli connecting $\mathbf{a}$ to $\mathbf{b}$ in the computation of $G \mathbf{a b}$ may be replaced by continuous paths. One prominent class of stimulus spaces in which this happens is that of spaces with intermediate points. The defining property of such a space is that with any two distinct points $\mathbf{a}, \mathbf{b}$ it contains a third distinct point $\mathbf{m}$ such that

$$
D \mathbf{a m}+D \mathbf{m b} \leq D \mathbf{a b} .
$$

If $D$ is a metric, this notion specializes to that of a convex space in the sense of Menger, where the inequality is replaced with equality,

$$
D \mathbf{a m}+D \mathbf{m b}=D \mathbf{a b} .
$$


If a space with intermediate points is also complete (as defined in Section 2.3), the oriented metric Gab induced by $D$ is intrinsic: $G \mathbf{a b}$ coincides with the infimum of $D$-lengths of all arcs connecting $\mathbf{a}$ to $\mathbf{b}$.

In UFS, all computations of the path length theory are invariant with respect to which of the two canonical psychometric increments, $\Psi^{(1)}$ or $\Psi^{(2)}$, is chosen to play the role of $D$.
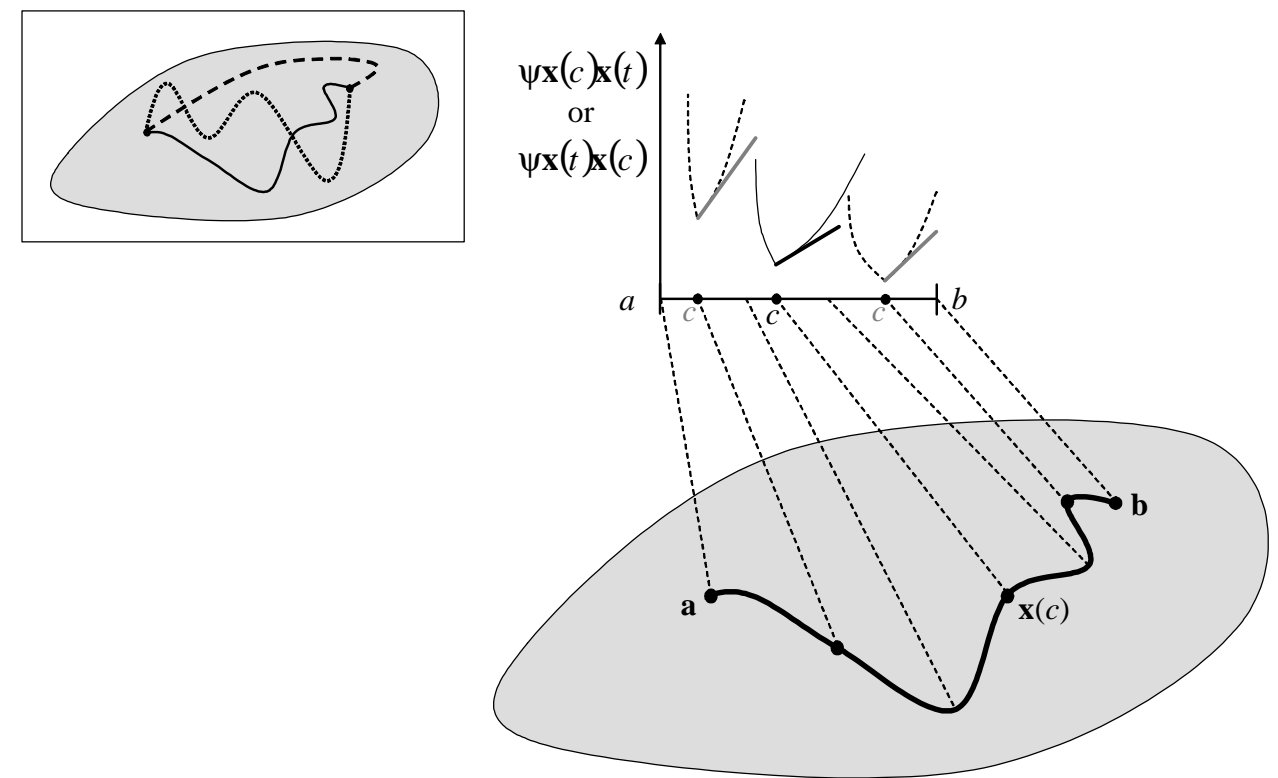

Figure 4. A continuously differentiable path $\mathbf{x}(t)$ (thick curve) is shown as a mapping of an interval $[a, b]$ (horizontal line segment) into a stimulus space (grey area). For any point $c \in[a, b]$ there is a function $t \mapsto \psi \mathbf{x}(c) \mathbf{x}(t)$ defined for all $t \in[a, b]$ (shown by $V$-shaped curves for three positions of $c$ ). The derivative of $\psi \mathbf{x}(c) \mathbf{x}(t)$ at $t=c+$ (the slope of the tangent line at the minimum of the $V$-shaped curve) is taken for the value of $F_{1}(\mathbf{x}(c), \dot{\mathbf{x}}(c))$, and the integral of this function from $a$ to $b$ is taken for the value of $\Psi^{(1)}$-length of the path $\left(\Psi^{(1)}\right.$ playing the role of dissimilarity $\left.D\right)$. The $\Psi^{(2)}$-length of the path is defined analogously, by integrating $F_{2}(\mathbf{x}(c), \dot{\mathbf{x}}(c))$ which is obtained by differentiating $\psi \mathbf{x}(t) \mathbf{x}(c)$ at $t=c+$. The inset at the left top corner shows that one should consider the $\Psi^{(1)}$-lengths (or $\Psi^{(2)}$-lengths) for all such paths from $\mathbf{a}$ to $\mathbf{b}$, and take their infimum as the oriented distance $G_{1} \mathbf{a b}$ (resp., $G_{2} \mathbf{a b}$ ). The overall, symmetric distance $G^{*} \mathbf{a b}$ is computed as $G_{1} \mathbf{a b}+G_{1} \mathbf{b a}$ or $G_{2} \mathbf{a b}+G_{2} \mathbf{b a}$, the two sums being the same.

\subsection{Finslerian Geometry of Path Length (Informal Account)}

The motivation for the present paper is related to that fact that the treatment of path length and intrinsic metrics in Dzhafarov (2007) is quite different from the differential-geometric treatment with which the theoretical program of Generalized Fechnerian Scaling began (Dzhafarov, 2002a-d; Dzhafarov \& Colonius, 1999, 2001). The differential-geometric treatment is closer to our interpretation of Fechner's original theory (Fig. 1) in that the dissimilarity cumulation in it is effected by integrating dissimilarities between "infinitesimally 
close" stimuli.

The scope of this differential-geometric treatment is restricted to stimulus spaces representable canonically (i.e., with (3) satisfied) by open connected regions of Euclidean $n$-space. Any two points $\mathbf{a}, \mathbf{b}$ in such a space can be connected by a continuously differentiable path $\mathbf{x}(t)$ defined on a segment of reals $[a, b]$ (see Fig. 4). Its $D$-length (with $D$ standing for either $\Psi^{(1)}$ or $\Psi^{(2)}$ ) can be defined by means of the following construction. Suppose that for any $c \in[a, b]$ the discrimination probabilities $\psi \mathbf{x}(c) \mathbf{x}(t)$ and $\psi \mathbf{x}(t) \mathbf{x}(c)$ have positive right-hand derivatives at $t=c+$,

$$
\begin{gathered}
\left.\frac{\mathrm{d} \psi \mathbf{x}(c) \mathbf{x}(t)}{\mathrm{d} t}\right|_{t=c+}=\lim _{s \rightarrow 0+} \frac{\Psi^{(1)} \mathbf{x}(c) \mathbf{x}(c+s)}{s}=F_{1}(\mathbf{x}(c), \dot{\mathbf{x}}(c)) \\
\left.\frac{\mathrm{d} \psi \mathbf{x}(t) \mathbf{x}(c)}{\mathrm{d} t}\right|_{t=c+}=\lim _{s \rightarrow 0+} \frac{\Psi^{(2)} \mathbf{x}(c) \mathbf{x}(c+s)}{s}=F_{2}(\mathbf{x}(c), \dot{\mathbf{x}}(c)) .
\end{gathered}
$$

We term $F_{1}(\mathbf{x}(c), \dot{\mathbf{x}}(c))$ and $F_{2}(\mathbf{x}(c), \dot{\mathbf{x}}(c))$ submetric functions ${ }^{4}$ of the first and second kind. Assuming further that $F_{1}$ and $F_{2}$ are continuous, we define the $\Psi^{(1)}$-length and $\Psi^{(2)}$-length of the path $\mathbf{x}(t)$ as integrals

$$
\begin{aligned}
\Psi^{(1)}(\mathbf{x}[a, b]) & =\int_{a}^{b} F_{1}(\mathbf{x}(t), \dot{\mathbf{x}}(t)) \mathrm{d} t \\
\Psi^{(2)}(\mathbf{x}[a, b]) & =\int_{a}^{b} F_{2}(\mathbf{x}(t), \dot{\mathbf{x}}(t)) \mathrm{d} t .
\end{aligned}
$$

Applying this to all continuously differentiable paths connecting $\mathbf{a}$ to $\mathbf{b}$ and finding the infima of their $\Psi^{(1)}{ }_{-}$ lengths and $\Psi^{(2)}$-lengths, one defines the oriented Fechnerian distances from $\mathbf{a}$ to $\mathbf{b}$ of the first and second kind, $G_{1} \mathbf{a b}$ and $G_{2} \mathbf{a b}$. The overall Fechnerian distance $G^{*} \mathbf{a b}$ is computed by the symmetrization scheme (1), yielding the same result whether one uses $G_{1}$ derived from $\Psi^{(1)}$ or $G_{2}$ derived from $\Psi^{(2)}$ (Dzhafarov, 2002d; Dzhafarov \& Colonius, 2005a).

Mathematically, the oriented metrics $G_{1}$ and $G_{2}$ are known as Finsler metrics in the broad sense of the term (Dzhafarov \& Colonius, 1999, 2001). ${ }^{5}$ Riemannian metrics being a subclass of Finsler ones, one can, with some additional assumptions, find this approach to subjective distances in the derivation of color metrics from color discrimination functions (Indow, 1994; Robertson, 1978; Wyszecki \& Stiles, 1982) dating back to the classical work of Helmholtz (1891) and Schrödinger (1920, 1920/1970, 1926/1970). One can also relate this approach, somewhat less directly, to the differential-geometric constructions considered in Townsend, Aisbett, Busemeyer, and Assadi (2006), Townsend, Solomon, and Spencer-Smith (2001), and Zhang $(2004,2006)$, as well as to the "dimensionality reduction" techniques proposed in Roweis and Saul (2000) and Tenenbaum, de Silva, and Langford (2000), dating back to Shepard and Carroll (1966).

\footnotetext{
${ }^{4}$ In Dzhafarov \& Colonius (2005a) we changed the traditional differential-geometric term metric function into submetric function, to avoid confusing it with a function which happens to be a metric (distance function).

${ }^{5}$ The "broad sense" means that the submetric function $F(\mathbf{x}(t), \dot{\mathbf{x}}(t))$, omitting indices, is positive, continuous, and first-order homogeneous in $\dot{\mathbf{x}}(t)$ (see Theorem 11 in Section 6 for precise formulations), ensuring thereby that $\Psi$ (x $[a, b]$ ) is well-defined and invariant under diffeomorphic reparametrizations of paths. Finsler geometry in the narrow sense requires in addition that $F(\mathbf{x}(t), \dot{\mathbf{x}}(t))$ be differentiable sufficient (e.g., infinite) number of times in the components of both $\mathbf{x}(t)$ and $\dot{\mathbf{x}}(t)$, and that the second-order derivatives of $F^{2}(\mathbf{x}(t), \dot{\mathbf{x}}(t))$ with respect to the components of $\dot{\mathbf{x}}(t)$ form a positive-definite matrix (Shen, 2001). Intermediate definitions can be obtained, e.g., by imposing convexity or strict convexity conditions on the indicatrices $\mathfrak{I}_{\mathbf{a}}=\{\mathbf{x}: F(\mathbf{a}, \mathbf{x}-\mathbf{a}) \leq 1\}$ (Busemann, 1950, 2005).
} 
In the unidimensional context, when stimuli are represented by real numbers (such as weight or intensity measurements), the notion of a path is superfluous. Nevertheless, the differential-geometric scheme described above formally applies here too, by taking, for instance, the identity mappings $[a, b] \mapsto[a, b]$ as unidimensional paths. This allows one to obtain a certain variant of Fechner's original theory as a proper special case of the Finslerian treatment, with $n=1$. Under the additional assumption that an increase in the magnitude of stimulus corresponds to an increase in some semantically unidimensional attribute ("sensation"), the slopes of the probability-of-different functions in (6) and Fig. 4 can be replaced by the slopes of the probability-ofgreater functions at their medians. Our interpretation of Fechner's theory then will agree with that proposed by Pfanzagl (1962); variants or elaborations of Pfanzagl's interpretation can be found in Creelman (1967), Falmagne (1971), Krantz (1971), and Iverson (2006).

\subsection{Building Bridges}

The differential-geometric (Finslerian) theory for path length and Fechnerian metrics, if valid, should constitute a special case of the general theory developed in Dzhafarov (2007) for path-connected spaces. The relationship between the two, however, is far from being trivial. The main purpose of the present paper is to show how the Finslerian theory can be achieved by a specialization, through intermediate constructions which are of interest in their own right, of the dissimilarity-based path length theory. These intermediate constructions, like the general theory of UFS, are purely psychological, in the technical sense of Dzhafarov and Colonius (2005a, b): when dissimilarity $D$ is specified as $\Psi^{(1)}$ or $\Psi^{(2)}$ in a stimulus space, every stimulus a is entirely characterized by the probabilities of its discrimination from other stimuli, whereby all computations of the theory are invariant under all possible relabelings (bijective transformations) of the stimuli.

The Finslerian theory, by contrast, is psycho-physical (again, in the technical sense of Dzhafarov \& Colonius, 2005a, b): it makes use of the physical properties of stimulus space, such as dimensionality, vectorial structure, and Euclidean topology. There are probably many reasonable ways of arriving at such a theory. The variant we choose is more general than the Multidimensional Fechnerian Scaling (MDFS) which started our theoretical program (Dzhafarov \& Colonius, 2001), except for one aspect in which the present theory is more restrictive: as it turns out, the computation of the path length by means of the integration of a submetric function only accords with the general theory of path length if the submetric function is convex (a routine assumption in mathematical theories of Finsler spaces which however we found unnecessary to posit in Dzhafarov \& Colonius, 2001, and subsequent publications).

The development to be presented, being derived from a new approach to dissimilarity and subjective distance (the DC theory), is different in many essential respects from our earlier generalization of the Multidimensional Fechnerian Scaling in Dzhafarov and Colonius (2005a). 


\subsection{Plan of the Paper}

In the next section we recapitulate the notions and results from Dzhafarov and Colonius (2007) and Dzhafarov (2007) which we need for the present development. In Section 3 we introduce a definition of a smooth path and establish its properties. In Section 4 we study stimulus spaces in which dissimilarities can be approximated by lengths of special smooth arcs, called simple. In Section 5 we introduce a general notion of the dissimilarity function that acts locally as a metric, and consider simple arcs that act as geodesics when connecting "very close" points. In Section 6 we introduce axioms linking the purely psychological notions of the previous sections with the basic properties of Euclidean $n$-space when the latter serves to represent stimuli. In Section 7 we look at details of how the abstract notion of dissimilarity $D$ can be replaced with psychometric increments $\Psi^{(1)}$ and $\Psi^{(2)}$. The concluding section of the paper provides a brief summary of our results and a general overview of the DC-UFS theory.

\section{Some Definition and Results from the Previous Development}

\subsection{Notation}

As should be apparent from the foregoing, we use boldface lowercase letters to denote stimuli (usually referred to as points), $\mathbf{a}, \mathbf{b}^{\prime}, \mathbf{x}, \mathbf{y}_{n}$, etc. Sets of stimuli are denoted by Gothic letters, $\mathfrak{S}, \mathfrak{S}_{1}, \mathfrak{A}$, etc.

Finite chains of stimuli are presented as strings of points, $\mathbf{x}_{1} \ldots \mathbf{x}_{k}, k \geq 0$ being referred to as the chain's cardinality. A chain as a whole is denoted by an uppercase boldface letter, $\mathbf{X}, \mathbf{Y}_{n}$, etc. If $\mathbf{X}=\mathbf{x}_{1} \ldots \mathbf{x}_{k}$, $\mathbf{Y}=\mathbf{y}_{1} \ldots \mathbf{y}_{l}$, then $\mathbf{X Y}=\mathbf{x}_{1} \ldots \mathbf{x}_{k} \mathbf{y}_{1} \ldots \mathbf{y}_{l}$, appropriately renumbered. In particular, $\mathbf{a X b}$ is a chain connecting a to $\mathbf{b}$.

A real-valued function of two or more stimuli is indicated by a symbol for the function followed by a string of stimuli: $\psi \mathbf{a b}, D \mathbf{a b c}, D \mathbf{X}_{n}, \Psi^{(\iota)} \mathbf{a b}$, etc.

For $\mathbf{X}=\mathbf{x}_{1} \ldots \mathbf{x}_{k}$, the expression $D \mathbf{x}_{1} \ldots \mathbf{x}_{k}$, or $D \mathbf{X}$ (and analogous expressions with $\Psi^{(1)}$ and $\Psi^{(2)}$ playing the role of $D$ ), is always understood as the cumulated dissimilarity along the chain $\mathbf{x}_{1} \ldots \mathbf{x}_{k}$,

$$
D \mathbf{X}=D \mathbf{x}_{1} \mathbf{x}_{2}+D \mathbf{x}_{2} \mathbf{x}_{3}+\ldots+D \mathbf{x}_{k-1} \mathbf{x}_{k}
$$

To distinguish a function $\mathbf{x}: A \mapsto \mathfrak{S}$ from a point $\mathbf{x}$ the former is always indicated with its domain, $\mathbf{x} \mid A$. In particular, a path $\mathbf{x}:[a, b] \mapsto \mathfrak{S}$ is denoted $\mathbf{x} \mid[a, b]$. If $B \subset A$, the restriction of $\mathbf{x} \mid A$ to $B$ is denoted $\mathbf{x} \mid B{ }^{6}$

We use the square-bracket notation for intervals of reals (closed, open and half-open): $[a, b],[a, b[] a, b$,$] ,$ ]$a, b[$. So $(a, b)$ always indicates an ordered pair of numbers.

Other notation conventions will be explained as they are introduced.

\footnotetext{
${ }^{6}$ In Dzhafarov (2007) paths were usually denoted by letters $\mathbf{f}, \mathbf{g}$, and $\mathbf{h}$ to distinguish them from "free" points $\mathbf{a}, \mathbf{b}, \mathbf{x}, \mathbf{y}$, etc. Using the same symbols for paths and points is more in a differential-geometric tradition.
} 


\subsection{Dissimilarity, Chains, Distance}

Stimuli are assumed to belong to a set $\mathfrak{S}$ endowed with a discrimination probability function $\psi: \mathfrak{S} \times \mathfrak{S} \mapsto$ $[0,1]$ subject to following constraints:

$$
\begin{gathered}
\psi \mathbf{a y}=\psi \mathbf{b y}, \text { for all } \mathbf{y} \in \mathfrak{S} \Longrightarrow \mathbf{a}=\mathbf{b} \\
\psi \mathbf{x a}=\psi \mathbf{x b}, \text { for all } \mathbf{x} \in \mathfrak{S} \Longrightarrow \mathbf{a}=\mathbf{b} \\
\mathbf{a} \neq \mathbf{b} \Longrightarrow \psi \mathbf{a}<\min \{\psi \mathbf{a b}, \psi \mathbf{b} \mathbf{a}\}
\end{gathered}
$$

In the complete theory (Dzhafarov \& Colonius, 2007) the discrimination probability function need not have these properties, but the properties it is postulated to have allow one to relabel the stimuli so that (9) is satisfied. The first two properties in (9) encapsulate the principle of the purely psychological description: two stimuli which behave identically with respect to all stimuli they are compared to are assigned identical labels. Once this has been done, the third property in (9) is a consequence of the law of Regular Minimality: for every stimulus one can find a stimulus it is least discriminable from, and the relation of being least discriminable is symmetrical ( $\mathbf{a}$ is least discriminable from $\mathbf{b}$ if and only if $\mathbf{b}$ is least discriminable from $\mathbf{a}$ ). In the canonical stimulus space $(\mathfrak{S}, \psi)$ the pairs of stimuli which are least discriminable from each other are assigned identical labels (see footnote 3). The third property in (9) therefore is referred to as the law of Regular Minimality in the canonical form. It guarantees that the canonical psychometric increments of the first and second kind, $\Psi^{(1)} \mathbf{a b}$ and $\Psi^{(2)} \mathbf{a b}$ in (3), are positive for all distinct $\mathbf{a}, \mathbf{b}$. (In the following the adjective canonical is sometimes omitted for brevity. $)^{7}$

We postulate that both $\Psi^{(1)}$ and $\Psi^{(2)}$ are dissimilarity functions. Denoting either of them by $D$, the (uniform) dissimilarity function is defined by the following properties:

D1. $\mathbf{a} \neq \mathbf{b} \Longrightarrow D \mathbf{a b}>0$

D2. $D \mathbf{a a}=0$

D3. (Uniform Continuity) If $D \mathbf{a}_{n} \mathbf{a}_{n}^{\prime} \rightarrow 0$ and $D \mathbf{b}_{n} \mathbf{b}_{n}^{\prime} \rightarrow 0$, then $D \mathbf{a}_{n}^{\prime} \mathbf{b}_{n}^{\prime}-D \mathbf{a}_{n} \mathbf{b}_{n} \rightarrow 0$.

\footnotetext{
${ }^{7}$ Note that (9) contains in it an empirical hypothesis, according to which $\psi \mathbf{a b}$ may only equal $\psi \mathbf{a a}$ if $\mathbf{a}$ and $\mathbf{b}$ identically compare to all stimuli in the stimulus space: $\psi \mathbf{a y}=\psi \mathbf{b y}$ for all $\mathbf{y}$ and $\psi \mathbf{x a}=\psi \mathbf{x b}$ for all $\mathbf{x}$ - implying that $\mathbf{a}$ and $\mathbf{b}$ are psychologically equal and should be assigned identical labels. Conversely, if $\psi \mathbf{a y} \neq \psi \mathbf{b y}$ for a single $\mathbf{y}$ or $\psi \mathbf{x a} \neq \psi \mathbf{x b}$ for a single $\mathbf{x}$, it should be assumed that $\mathbf{a}$ and $\mathbf{b}$ have different percepts, whence $\psi \mathbf{a b}$ (the probability with which $\mathbf{a}$ and $\mathbf{b}$ are judged to be different) must exceed both $\psi \mathbf{a a}$ and $\psi \mathbf{b b}$. It is easy to verify that (9) does not allow for the perceptual version of the ancient Greek "sorites" paradox - the hypothetical intransitivity of the perceptual equality relation: a is indistinguishable from $\mathbf{b}$ (presumably meaning $\psi \mathbf{a a}=\psi \mathbf{a b}=\psi \mathbf{b} \mathbf{a}=\psi \mathbf{b} \mathbf{b})$, $\mathbf{b}$ is indistinguishable from $\mathbf{c}(\psi \mathbf{b} \mathbf{b}=\psi \mathbf{b} \mathbf{c}=\psi \mathbf{c b}=\psi \mathbf{c c})$, but $\mathbf{a}$ is distinguishable from $\mathbf{c}(\psi \mathbf{a a}<\psi \mathbf{a c}$, or $\psi \mathbf{a a}<\psi \mathbf{c a})$. There seems to be no factual evidence of these probabilistic relations ever happening: the intransitivity is only alleged if the (in)distinguishability is described in terms of "just noticeable differences," ignoring their probabilistic nature. This is of course an incomplete account of the perceptual "sorites." A detailed one should consider that $\mathbf{a}$ and $\mathbf{b}$ in $\psi \mathbf{a b}$ belong to different observation areas (see footnote 3), whereas in statements $\psi \mathbf{a y}=\psi \mathbf{b y}$ and $\psi \mathbf{x a}=\psi \mathbf{x b}$ they belong to one and the same observation area (Dzhafarov \& Colonius, 2007). One should also consider such issues as physical variability of stimuli being compared (Dzhafarov, 2006) and the possibility of "creative interactions" between their images. Such a discussion, however, would lead us beyond the scope of this paper.
} 
D4. for any sequence of chains $\mathbf{a}_{n} \mathbf{X}_{n} \mathbf{b}_{n}$,

$$
D \mathbf{a}_{n} \mathbf{X}_{n} \mathbf{b}_{n} \rightarrow 0 \Longrightarrow D \mathbf{a}_{n} \mathbf{b}_{n} \rightarrow 0
$$

In accordance with our notation agreements, if $\mathbf{X}_{n}=\mathbf{x}_{1}^{n} \ldots \mathbf{x}_{k_{n}}^{n}$,

$$
D \mathbf{a}_{n} \mathbf{X}_{n} \mathbf{b}_{n}=D \mathbf{a}_{n} \mathbf{x}_{1}^{n}+\sum_{i=1}^{k-1} D \mathbf{x}_{i}^{n} \mathbf{x}_{i+1}^{n}+D \mathbf{x}_{k}^{n} \mathbf{b}_{n}
$$

A conventional (symmetric) metric is always a dissimilarity function, but a dissimilarity generally is not symmetric and does not satisfy the triangle inequality.

The following results and definitions are taken from Dzhafarov and Colonius (2007), which should be consulted for details, including reminders for general mathematical terms (topological base, uniformity, etc.).

We define the convergence $\mathbf{a}_{n} \leftrightarrow \mathbf{b}_{n}$ in the stimulus space as meaning $D \mathbf{a}_{n} \mathbf{b}_{n} \rightarrow 0$.

Proposition 1 The convergence $\leftrightarrow$ is reflexive, symmetric, and transitive.

Proposition $2 D$ induces on $\mathfrak{S}$ a topology and a uniformity. The topology is based on open balls

$$
\mathfrak{B}_{D}(\mathbf{x}, \varepsilon)=\{\mathbf{y} \in \mathfrak{S}: D \mathbf{x y}<\varepsilon\}
$$

taken for all $\mathbf{x} \in \mathfrak{S}$ and all real $\varepsilon>0$. The uniformity is based on entourages

$$
\mathfrak{U}_{D}(\varepsilon)=\left\{(\mathbf{x}, \mathbf{y}) \in \mathfrak{S}^{2}: D \mathbf{x y}<\varepsilon\right\}
$$

taken for all real $\varepsilon>0$.

We define the Fechnerian metrics $G$ and $G^{*}$ by

$$
\begin{gathered}
G \mathbf{a b}=\inf _{\text {all possible } \mathbf{x}} D \mathbf{a X b}, \\
G^{*} \mathbf{a b}=G \mathbf{a b}+G \mathbf{b a} .
\end{gathered}
$$

Proposition $3 G^{*}$ is a symmetric metric (called the "overall" Fechnerian metric), hence $G^{*}$ is a dissimilarity function. $G$ is an oriented metric which is also a dissimilarity function. The topology (uniformity) induced on $\mathfrak{S}$ by $G$ coincides with the topology (uniformity) induced on $\mathfrak{S}$ by $D$.

Proposition $4 G \mathbf{a b}$ is uniformly continuous in $(\mathbf{a}, \mathbf{b})$, i.e., if $\mathbf{a}_{n}^{\prime} \leftrightarrow \mathbf{a}_{n}$ and $\mathbf{b}_{n}^{\prime} \leftrightarrow \mathbf{b}_{n}$, then $G \mathbf{a}_{n}^{\prime} \mathbf{b}_{n}^{\prime}-$ $G \mathbf{a}_{n} \mathbf{b}_{n} \rightarrow 0$.

In UFS, Dab is specified to be $\Psi^{(1)} \mathbf{a b}=\psi \mathbf{a b}-\psi \mathbf{a} \mathbf{a}$ or $\Psi^{(2)} \mathbf{a b}=\psi \mathbf{b} \mathbf{a}-\psi \mathbf{a} \mathbf{a}$.

Proposition $5 \Psi^{(1)} \mathbf{a}_{n} \mathbf{b}_{n} \rightarrow 0$ if and only if $\Psi^{(2)} \mathbf{a}_{n} \mathbf{b}_{n} \rightarrow 0$ (i.e., both mean $\mathbf{a}_{n} \leftrightarrow \mathbf{b}_{n}$ ).

Proposition 6 The (canonical) discrimination probability function $\psi \mathbf{a b}$ is uniformly continuous: if $\mathbf{a}_{n}^{\prime} \leftrightarrow$ $\mathbf{a}_{n}$ and $\mathbf{b}_{n}^{\prime} \leftrightarrow \mathbf{b}_{n}$, then $\psi \mathbf{a}_{n}^{\prime} \mathbf{b}_{n}^{\prime}-\psi \mathbf{a}_{n} \mathbf{b}_{n} \rightarrow 0$. 
Replacing $D$ in (10) with $\Psi^{(1)}$ and $\Psi^{(2)}$ we get two oriented metrics,

$$
\begin{aligned}
& G_{1} \mathbf{a b}=\inf _{\mathbf{X}} \Psi^{(1)} \mathbf{a} \mathbf{X b}, \\
& G_{2} \mathbf{a b}=\inf _{\mathbf{X}} \Psi^{(2)} \mathbf{a X} \mathbf{b} .
\end{aligned}
$$

Proposition 7 For any $\mathbf{a}, \mathbf{b}$,

$$
G_{1} \mathbf{a b}-G_{2} \mathbf{b a}=G_{2} \mathbf{a b}-G_{1} \mathbf{b a}=\psi \mathbf{b} \mathbf{b}-\psi \mathbf{a a},
$$

whence

$$
G_{1} \mathbf{a b}+G_{1} \mathbf{b a}=G_{2} \mathbf{a b}+G_{2} \mathbf{b a}=G^{*} \mathbf{a b} .
$$

That is, the overall Fechnerian metric $G^{*}$ ab is the same for the two kinds of psychometric increments.

The latter property provides the main justification for the addition of the oriented distances "to and from" $\left(G_{1} \mathbf{a b}+G_{1} \mathbf{b a}, G_{2} \mathbf{a b}+G_{2} \mathbf{b a}\right)$ : as shown in Dzhafarov and Colonius (2007), it is satisfied by no other "reasonable" symmetrization scheme applicable to all possible spaces satisfying (9).

\subsection{Paths, Arcs, and their Lengths}

We now recapitulate some definitions and results from Dzhafarov (2007). Properties D1-D4 are the only assumptions posited for the dissimilarity space $(\mathfrak{S}, D)$, and these assumptions impose on $(\mathfrak{S}, D)$ topological and uniform structures (Proposition 2). In particular, since the notion of (uniform) convergence $\mathbf{a}_{n} \leftrightarrow \mathbf{b}_{n}$ in the space $(\mathfrak{S}, D)$ is well-defined, we can meaningfully speak of continuous and uniformly continuous functions from reals into $\mathfrak{S}$. A path is a continuous (hence uniformly continuous) mapping $\mathbf{x}:[a, b] \mapsto \mathfrak{S}$, or $\mathbf{x} \mid[a, b]$.

Given a net

$$
\mu=\left(a=x_{0} \leq x_{1} \leq \ldots \leq x_{k} \leq x_{k+1}=b\right)
$$

partitioning $[a, b]$, and a chain

$$
\mathbf{X}=\mathbf{x}_{0} \mathbf{x}_{1} \ldots \mathbf{x}_{k} \mathbf{x}_{k+1}
$$

of the same cardinality, the chain-on-net $\mathbf{X}^{\mu}$ is defined as

$$
\mathbf{X}^{\mu}=\left(\left(x_{0}, \mathbf{x}_{0}\right),\left(x_{1}, \mathbf{x}_{1}\right), \ldots,\left(x_{k}, \mathbf{x}_{k}\right),\left(x_{k+1}, \mathbf{x}_{k+1}\right)\right) .
$$

We also define

$$
\begin{array}{cc}
\delta \mu=\max _{i=0,1 \ldots, k}\left(x_{i+1}-x_{i}\right) & \text { (mesh of the net) } \\
\sigma_{\mathbf{x} \mid[a, b]}\left(\mathbf{X}^{\mu}\right)=\max _{i=0,1 \ldots, k} D \mathbf{x}\left(x_{i}\right) \mathbf{x}_{i} . & \left(\text { separation of } \mathbf{X}^{\mu} \text { from } \mathbf{x} \mid[a, b]\right)
\end{array},
$$

and the convergence of $\mathbf{X}^{\mu}$ to $\mathbf{x} \mid[a, b]$,

$$
\left.\mathbf{X}^{\mu} \rightarrow \mathbf{x} \mid[a, b]\right) \Longleftrightarrow \delta \mu \rightarrow 0 \text { and } \sigma_{\mathbf{x} \mid[a, b]}\left(\mathbf{X}^{\mu}\right) \rightarrow 0
$$


The $D$-length of path $\mathbf{x} \mid[a, b]$, denoted $D \mathbf{x}([a, b]),{ }^{8}$ is defined as

$$
D \mathbf{x}([a, b])=\liminf _{\substack{\mathbf{X}^{\mu} \in \mathcal{M}_{a}^{b} \\ \mathbf{X}^{\mu} \rightarrow \mathbf{x} \mid[a, b]}} D \mathbf{X}=\liminf _{\substack{\left.\mathbf{X}^{\mu} \in \mathcal{M}_{a}^{b} \\ \delta \mu \rightarrow 0 \\ \sigma_{\mathbf{x} \mid[a, b]} \rightarrow \mathbf{X}^{\mu}\right) \rightarrow 0}} D \mathbf{X}
$$

where $\mathcal{M}_{a}^{b}$ is the set of all chains-on-nets whose nets partition $[a, b]$.

Proposition 8 For any path $\mathbf{x} \mid[a, b]$ connecting $\mathbf{a}$ to $\mathbf{b}$,

$$
D \mathbf{x}([a, b]) \geq G \mathbf{a b} \geq 0,
$$

and $D \mathbf{x}([a, b])=0$ if and only if $\mathbf{x}([a, b])=\{\mathbf{a}\}=\{\mathbf{b}\}$.

Proposition $9 D$-length is additive, i.e., for any $c \in[a, b]$,

$$
D \mathbf{x}([a, c])+D \mathbf{x}([c, b])=D \mathbf{x}([a, b]) .
$$

For $[x, y] \subset[a, b], D \mathbf{x}([x, y])$ is uniformly continuous in $(x, y)$, nondecreasing in $y$ and nonincreasing in $x$.

A sequence of paths $\mathbf{x}_{n} \mid[a, b]$ is said to converge to a path $\mathbf{x} \mid[a, b]$, in symbols $\mathbf{x}_{n}|[a, b] \rightarrow \mathbf{x}|[a, b]$, if

$$
\sigma_{\mathbf{x} \mid[a, b]}\left(\mathbf{x}_{n} \mid[a, b]\right)=\max _{x \in[a, b]} D \mathbf{x}(x) \mathbf{x}_{n}(x) \rightarrow 0
$$

as $n \rightarrow \infty$.

Proposition 10 (Lower semicontinuity) For any $\mathbf{x}_{n}|[a, b] \rightarrow \mathbf{x}|[a, b]$

$$
D \mathbf{x}([a, b]) \leq \liminf _{n \rightarrow \infty} D \mathbf{x}_{n}([a, b]) .
$$

Two paths $\mathbf{x} \mid[a, b]$ and $\mathbf{y} \mid[c, d]$ are each others' reparametrizations if for some nondecreasing and onto (hence continuous) mapping $\phi:[c, d] \mapsto[a, b]$,

$$
\mathbf{y}(x)=\mathbf{x}(\phi(x)), \quad x \in[c, d]
$$

Proposition 11 If $\mathbf{x} \mid[a, b]$ and $\mathbf{y} \mid[c, d]$ are each others' reparametrizations,

$$
D \mathbf{x}([a, b])=D \mathbf{y}([c, d]) .
$$

$D \mathbf{x}([a, b])$ may be infinite. If it is finite, $\mathbf{x} \mid[a, b]$ is said to be $D$-rectifiable. In this case $\mathbf{x} \mid[a, b]$ allows for a special reparametrization called natural D-parametrization. Denoting $D_{0}=D \mathbf{x}([a, b])$, the mapping $\phi:[a, b] \mapsto\left[0, D_{0}\right]$ is defined by

$$
\phi(x)=D \mathbf{x}([a, x]),
$$

\footnotetext{
${ }^{8}$ Although the expression $\mathbf{x}([a, b])$ denotes the image of the path in $\mathfrak{S}$, the expression $D \mathbf{x}([a, b])$ must not be taken to imply that the $D$-length of a path only depends on its image in $\mathfrak{S}$ : it is simply more convenient to write $D \mathbf{x}([a, b])$ than the more correct $D \mathbf{x} \mid[a, b]$
} 
and the natural $D$-parametrization of $\mathbf{x} \mid[a, b]$ is the path $\mathbf{n} \mid\left[0, D_{0}\right]$ such that

$$
\mathbf{x}(x)=\mathbf{n}(\phi(x)), \quad x \in[a, b] .
$$

It has the property

$$
D \mathbf{n}([u, v])=v-u
$$

for any $[u, v] \subset\left[0, D_{0}\right]$.

A path is an arc if it can be reparametrized into a homeomorphic path. In other words, $\mathbf{y} \mid[c, d]$ is an arc if one can find a nondecreasing and onto (hence continuous) mapping $\phi:[c, d] \mapsto[a, b]$, such that it satisfies (16) for some one-to-one and continuous (hence homeomorphic) function $\mathbf{x} \mid[a, b]$. Put equivalently, a path $\mathbf{y} \mid[c, d]$ is an arc if and only if, for any $\mathbf{a} \in \mathbf{y}([c, d]), \mathbf{y}^{-1}(\{\mathbf{a}\})$ is an interval (necessarily closed) in $[c, d]$. If an arc is rectifiable, then its natural $D$-parametrization is a homeomorphism.

Proposition 12 Let $\mathbf{x} \mid[a, b]$ be a $D$-rectifiable path with distinct endpoints $\mathbf{a}, \mathbf{b}$. Then there is an $\operatorname{arc}$ $\mathbf{y} \mid[a, b]$ connecting $\mathbf{a}$ to $\mathbf{b}$ such that

$$
\mathbf{y}([a, b]) \subset \mathbf{x}([a, b])
$$

and

$$
D \mathbf{y}([a, b]) \leq D \mathbf{x}([a, b])
$$

where the inequality is strict if $\mathbf{x} \mid[a, b]$ is not an arc.

Given a path $\mathbf{x} \mid[a, b]$ connecting $\mathbf{a}$ to $\mathbf{b}$, a chain-on-net $\mathbf{X}^{\mu}$ in (11) is said to be inscribed in this path if

$$
\mathbf{x}_{i}=\mathbf{x}\left(x_{i}\right), \quad i=0,1, \ldots, k+1
$$

For inscribed $\mathbf{X}^{\mu} \in \mathcal{M}_{a}^{b}, \sigma_{\mathbf{x} \mid[a, b]}\left(\mathbf{X}^{\mu}\right)=0$, so

$$
\left.\mathbf{X}^{\mu} \rightarrow \mathbf{x} \mid[a, b]\right) \Longleftrightarrow \delta \mu \rightarrow 0
$$

Define

$$
D_{\text {ins }} \mathbf{X}([a, b])=\lim _{\substack{\text { inscribed } \mathbf{X}^{\mu} \in \mathcal{M}_{a}^{b} \\ \mathbf{X}^{\mu} \rightarrow \mathbf{x} \mid[a, b]}} D \mathbf{X}=\lim _{\substack{\text { inscribed } \mathbf{X}^{\mu} \in \mathcal{M}_{a}^{b} \\ \delta \mu \rightarrow 0}} D \mathbf{X} .
$$

This quantity need not exist, and if it does,

$$
D_{\text {ins }} \mathbf{x}([a, b]) \geq D \mathbf{x}([a, b])
$$

We have two important results related to inscribed chains-on-nets.

Proposition 13 If the dissimilarity $D$ is a metric (oriented or symmetric), then for any path $\mathbf{x} \mid[a, b]$,

$$
D \mathbf{x}([a, b])=D_{\text {ins }} \mathbf{x}([a, b])
$$

Proposition 13 will be generalized in Section 5. 
Proposition 14 Let $\mathbf{X}_{n}^{\nu_{n}}$ be a sequence of chains-on-nets inscribed in path $\mathbf{x} \mid[a, b]$, i.e.,

$$
\mathbf{X}_{n}^{\nu_{n}}=\left\{\left(x_{i}^{n}, \mathbf{x}\left(x_{i}^{n}\right)\right)\right\}_{i=0, \ldots, k_{n}+1} \quad\left(x_{0}^{n}=a, x_{k_{n}+1}^{n}=b\right) .
$$

Then there is a sequence of $\mathbf{Z}_{n}^{\mu_{n}} \rightarrow \mathbf{x} \mid[a, b]$ with $D \mathbf{Z}_{n} \rightarrow D \mathbf{x}([a, b])$ such that

$$
\mathbf{X}_{n}^{\nu_{n}} \subset \mathbf{Z}_{n}^{\mu_{n}}
$$

for every $n$.

In other words, if for every $n$ one chooses an arbitrary inscribed chain-on-net, then a sequence of $\mathbf{Z}_{n}^{\mu_{n}}$ converging to $\mathbf{x} \mid[a, b]$ pointwise and in length can be constructed so that each chain-on-net $\mathbf{Z}_{n}^{\mu_{n}}$ passes through the corresponding inscribed one (Fig. 5).

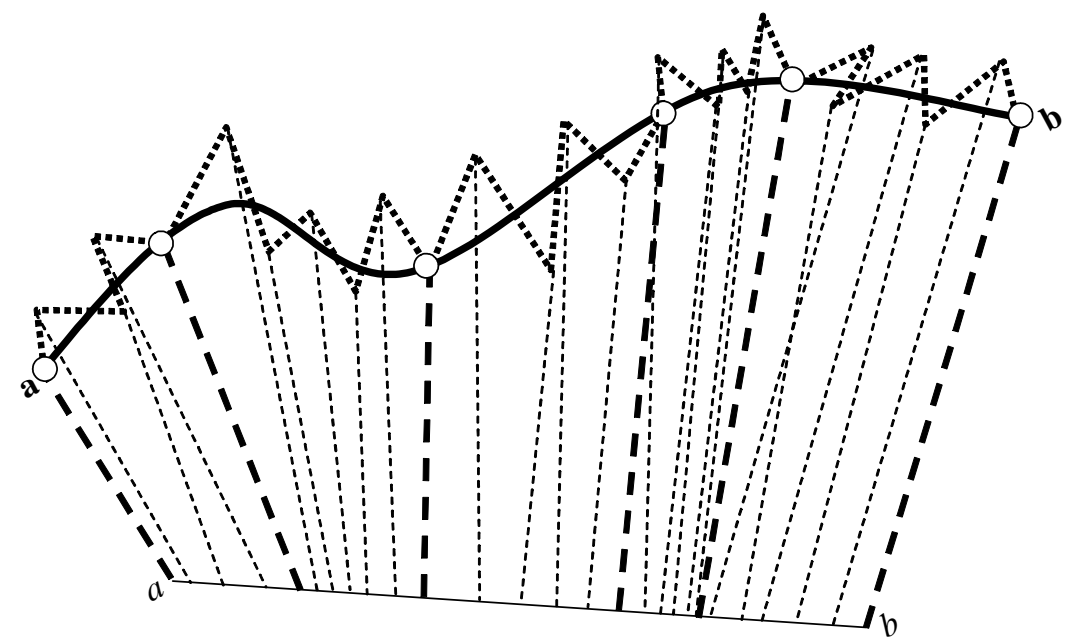

Figure 5. An illustration for Proposition 14. Open circles represent an inscribed chain (as they lie on the image of the path, shown by the thick curve). The chain shown by the point line "passes through" this inscribed chain (i.e., includes it as a subsequence).

Since the metric $G$ induced by $D$ in accordance with (10) is itself a dissimilarity function, the $G$-length of a path $\mathbf{x} \mid[a, b]$ is defined as

$$
G \mathbf{x}([a, b])=\liminf _{\substack{\mathbf{X}^{\mu} \in \mathcal{M}_{a}^{b} \\ \mathbf{X}^{\mu} \rightarrow \mathbf{x} \mid[a, b]}} G \mathbf{X}
$$

where (putting $\mathbf{X}=\mathbf{x}_{0} \mathbf{x}_{1} \ldots \mathbf{x}_{k} \mathbf{x}_{k+1}$ ),

$$
G \mathbf{X}=\sum_{i=0}^{k} G \mathbf{x}_{i} \mathbf{x}_{i+1} .
$$

(It is easy to show that the convergence $\mathbf{X}^{\mu} \rightarrow \mathbf{x} \mid[a, b]$ "in the $G$ sense" is the same as "in the $D$ sense.") The fundamental result here is that $G \mathbf{x}([a, b])$ and $D \mathbf{x}([a, b])$ always coincide.

Proposition 15 For any path $\mathbf{x} \mid[a, b]$,

$$
G \mathbf{x}([a, b])=D \mathbf{x}([a, b]) .
$$


The remaining topic from Dzhafarov (2007) we make use of in the present paper is that of intrinsic metrics and complete spaces with intermediate points. As mentioned in the Introduction, a space $(\mathfrak{S}, D)$ is said to be a space with intermediate points if for any distinct $\mathbf{a}, \mathbf{b}$ one can find an $\mathbf{m}$ such that $\mathbf{m} \notin\{\mathbf{a}, \mathbf{b}\}$ and $D \mathbf{a m b} \leq D \mathbf{a b}$. The space is complete if for any sequence of points $\mathbf{x}_{n}$ such that

$$
\lim _{\substack{k \rightarrow \infty \\ l \rightarrow \infty}} D \mathbf{x}_{k} \mathbf{x}_{l}=0
$$

there is a point $\mathbf{x}$ in $\mathfrak{S}$ such that $\mathbf{x}_{n} \leftrightarrow \mathbf{x}$.

Proposition 16 In a complete space with intermediate points, any $\mathbf{a}$ can be connected to any $\mathbf{b}$ by an arc $\mathbf{x} \mid[a, b]$ with

$$
G \mathbf{a b} \leq D \mathbf{x}([a, b]) \leq D \mathbf{a b}
$$

The Fechnerian metric $G \mathbf{a b}$ in such a space is intrinsic: $G \mathbf{a b}$ equals the infimum of $D \mathbf{x}([a, b])$ over all paths (equivalently, all arcs) connecting $\mathbf{a}$ to $\mathbf{b}$.

When applied to UFS, we have the following equivalence property which will be utilized in Section 7 .

Proposition $17\left(\mathfrak{S}, \Psi^{(1)}\right)$ is a complete space with intermediate points if and only if so is $\left(\mathfrak{S}, \Psi^{(2)}\right)$.

\section{Smooth paths}

We begin by introducing paths whose length can be obtained by integrating a generalized version of the submetric function $F(\mathbf{x}(t), \dot{\mathbf{x}}(t))$ mentioned in Section 1.3.

Refer to Fig. 6. A path $\mathbf{x} \mid[a, b]$ is called $D$-smooth (or simply smooth when confusion is unlikely) if

$$
\lim _{\tau-t \rightarrow 0+} \frac{D \mathbf{x}(t) \mathbf{x}(\tau)}{D \mathbf{x}([t, \tau])}=1
$$

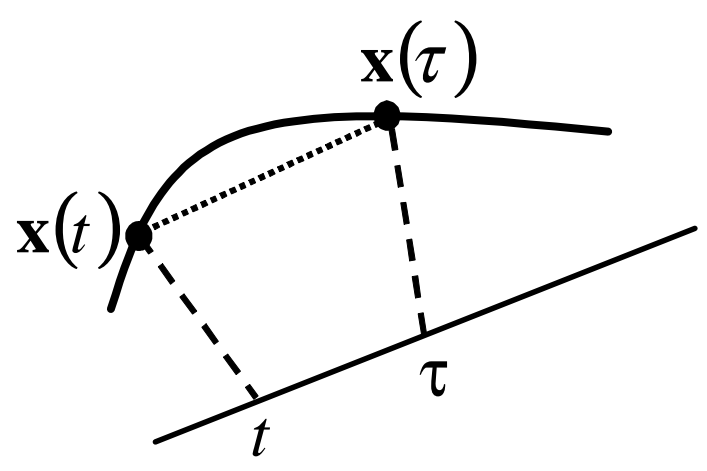

Figure 6. A fragment of a smooth path. As $t$ and $\tau$ get closer, the "chord-to-arc" ratio of the dissimilarity $D \mathbf{x}(t) \mathbf{x}(\tau)$ to the $D$-length from $\mathbf{x}(t)$ to $\mathbf{x}(\tau)$ tends to 1 . 
We could have formulated the definition in a seemingly weaker form: $\mathbf{x} \mid[a, b]$ is $D$-smooth if for any $c \in[a, b]$,

$$
\lim _{\substack{t \rightarrow c \\ \tau \rightarrow c}} \frac{D \mathbf{x}(t) \mathbf{x}(\tau)}{D \mathbf{x}([t, \tau])}=1
$$

It is easy to see, however, that the two definitions are equivalent.

Lemma 1 The definitions (24) and (25) are equivalent.

Proof. The implication $(24) \Longrightarrow(25)$ being obvious, we prove the reverse implication. Assume the contrary: let $(25)$ hold but there be a sequence $\left[t_{n}, \tau_{n}\right]$ with $\tau_{n}-t_{n} \rightarrow 0+$ and

$$
\frac{D \mathbf{x}\left(t_{n}\right) \mathbf{x}\left(\tau_{n}\right)}{D \mathbf{x}\left(\left[t_{n}, \tau_{n}\right]\right)} \nrightarrow 1 \text {. }
$$

Then either $\limsup$ or $\lim$ inf of the ratio tends to a quantity $L \neq 1$, and then for some subsequence of $\left[t_{n}, \tau_{n}\right]$ (without loss of generality, the sequence itself),

$$
\frac{D \mathbf{x}\left(t_{n}\right) \mathbf{x}\left(\tau_{n}\right)}{D \mathbf{x}\left(\left[t_{n}, \tau_{n}\right]\right)} \rightarrow L .
$$

The interval $[a, b]$ being compact, some subsequence of $\left[t_{n}, \tau_{n}\right] \subset[a, b]$ converges to a point $c \in[a, b]$, whence we arrive at a contradiction with (25).

Our first theorem says that even though $D$ is not a metric (compare with Proposition 13), when it comes to smooth paths,

$$
D \mathbf{x}([a, b])=D_{\text {ins }} \mathbf{x}([a, b]) .
$$

According to the definition of $D_{\text {ins }}$ in (21), this means that

$$
\lim _{n \rightarrow \infty} \sum_{i=0}^{k_{n}} D \mathbf{x}\left(t_{i}^{n}\right) \mathbf{x}\left(t_{i+1}^{n}\right)
$$

exists and equals $D \mathbf{x}([a, b])$ for any sequence of nets $\mu_{n}=\left(a=t_{0}^{n}, t_{1}^{n}, \ldots, t_{k_{n}}^{n}, t_{k_{n}+1}^{n}=b\right)$ with $\delta \mu_{n} \rightarrow 0$.

THEOREM 1 For a smooth path $\mathbf{x} \mid[a, b]$, and for any sequence of nets $\mu_{n}=\left(a=t_{0}^{n}, t_{1}^{n}, \ldots, t_{k_{n}}^{n}, t_{k_{n}+1}^{n}=b\right)$ with $\delta \mu_{n} \rightarrow 0$ as $n \rightarrow \infty$,

$$
D \mathbf{x}([a, b])=\lim _{n \rightarrow \infty} \sum_{i=0}^{k_{n}} D \mathbf{x}\left(t_{i}^{n}\right) \mathbf{x}\left(t_{i+1}^{n}\right)
$$

Proof. Since

$$
\lim _{\tau-t \rightarrow 0+} \frac{D \mathbf{x}(t) \mathbf{x}(\tau)}{D \mathbf{x}([t, \tau])}=1
$$

for every $\varepsilon>0$ one can find a $\delta>0$ such that $1-\varepsilon<\frac{D \mathbf{x}(t) \mathbf{x}(\tau)}{D \mathbf{x}([t, \tau])}<1+\varepsilon$ whenever $\tau-t<\delta$. Then, for any net $\mu_{n}$ with $\delta \mu_{n}<\delta$,

$$
1-\varepsilon<\frac{\sum_{i=0}^{k_{n}} D \mathbf{x}\left(t_{i}^{n}\right) \mathbf{x}\left(t_{i+1}^{n}\right)}{\sum_{i=0}^{k_{n}} D \mathbf{x}\left(\left[t_{i}^{n}, t_{i+1}^{n}\right]\right)}<1+\varepsilon
$$


But by the additivity of $D$-length (Proposition 9),

$$
\sum_{i=0}^{k_{n}} D \mathbf{x}\left(\left[t_{i}^{n}, t_{i+1}^{n}\right]\right)=D \mathbf{x}([a, b]),
$$

and the statement of the theorem follows.

The dissimilarities among points taken on a smooth path exhibit the following property that can be called additivity-in-the-small (Fig. 7).

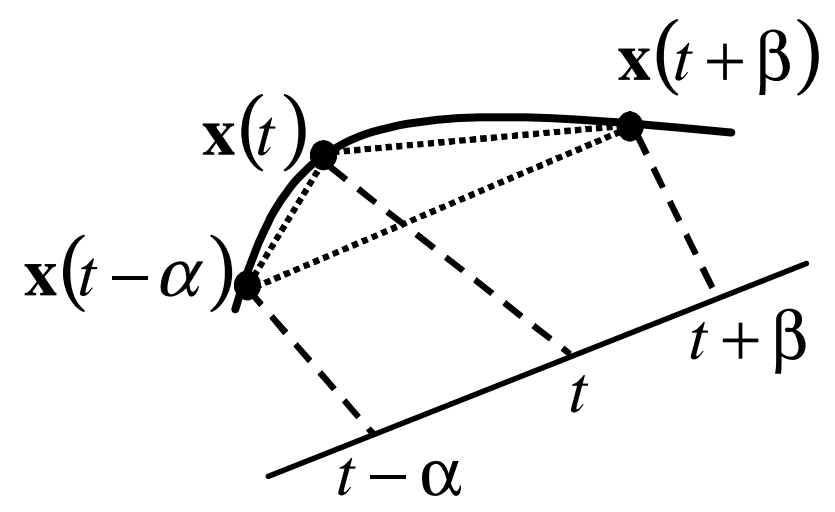

Figure 7. A fragment of a smooth path. As the two flanking points get closer to the fixed middle one, the sum of the dissimilarities $D \mathbf{x}(t-\alpha) \mathbf{x}(t)$ and $D \mathbf{x}(t) \mathbf{x}(t+\beta)$ gets closer to $D \mathbf{x}(t-\alpha) \mathbf{x}(t+\beta)$.

Theorem 2 For a smooth path $\mathbf{x} \mid[a, b]$ and any $t \in] a, b[$,

$$
\lim _{\substack{\alpha \rightarrow 0+\\ \beta \rightarrow 0+}} \frac{D \mathbf{x}(t-\alpha) \mathbf{x}(t)+D \mathbf{x}(t) \mathbf{x}(t+\beta)}{D \mathbf{x}(t-\alpha) \mathbf{x}(t+\beta)}=1
$$

Proof. On rewriting the ratio as

$$
\frac{D \mathbf{x}([t-\alpha, t]) \frac{D \mathbf{x}(t-\alpha) \mathbf{x}(t)}{D \mathbf{x}([t-\alpha, t])}+D \mathbf{x}([t, t+\beta]) \frac{D \mathbf{x}(t) \mathbf{x}(t+\beta)}{D \mathbf{x}([t, t+\beta])}}{D \mathbf{x}([t-\alpha, t+\beta]) \frac{D \mathbf{x}(t-\alpha) \mathbf{x}(t+\beta)}{D \mathbf{x}([t-\alpha, t+\beta])}}
$$

the result follows from the definition of a smooth path and the additivity property for $D$-length.

A path $\mathbf{x} \mid[a, b]$ is called smoothly D-parametrized if

$$
\lim _{s \rightarrow 0+} \frac{D \mathbf{x}(t) \mathbf{x}(t+s)}{s}
$$

exists and is positive and continuous in $t \in[a, b[$.

Clearly, the limit only depends on an arbitrarily small right-hand vicinity of $t$, and following Dzhafarov and Colonius (2005a) we denote this limit

$$
F(\mathbf{x}(t), \stackrel{\circ}{\mathbf{x}}(t))=\lim _{s \rightarrow 0+} \frac{D \mathbf{x}(t) \mathbf{x}(t+s)}{s}
$$


The composite symbol $(\mathbf{x}(t), \stackrel{\mathbf{x}}{(t)})$ is called an arc element, a generalization of the "line element" of a Finslerian geometry (see Section 6). Intuitively, an arc element indicates a point $\mathbf{x}(t)$ paired with a direction of stimulus change $\stackrel{\mathbf{x}}{(}(t)$, that can be thought of as an "infinitesimally small" arc. The formal definition given in Dzhafarov and Colonius (2005a) is as follows. Two paths $\mathbf{x} \mid[a, b]$ and $\mathbf{y} \mid[c, d]$ are called codirectional if $a=c$ and $\mathbf{x}(t)=\mathbf{y}(t)$ for all $t \in[a, \min \{b, d\}]$. Arc element $(\mathbf{x}(t), \stackrel{\circ}{\mathbf{x}}(t))$ is the equivalence class of all paths codirectional with $\mathbf{x} \mid[t, b](t \in[a, b[)$.

Given a path $\mathbf{x} \mid[a, b]$, an arc element $(\mathbf{x}(t), \stackrel{\circ}{\mathbf{x}}(t))$, and a $k>0$, the equivalence class of all paths codirectional with a fragment $\mathbf{y} \mid[t, t+s]$ of a path defined by $\mathbf{y}(t+s)=\mathbf{x}(t+k s)$ is an arc element $(\mathbf{y}(t), \mathbf{y}(t))$ which can be denoted $(\mathbf{x}(t), k \stackrel{\circ}{\mathbf{x}}(t))$. This notation and the observation that

$$
F(\mathbf{x}(t), k \stackrel{\circ}{\mathbf{x}}(t))=\lim _{s \rightarrow 0+} \frac{D \mathbf{x}(t) \mathbf{x}(t+k s)}{s}=k \lim _{k s \rightarrow 0+} \frac{D \mathbf{x}(t) \mathbf{x}(t+k s)}{k s}=k F(\mathbf{x}(t), \stackrel{\circ}{\mathbf{x}}(t))
$$

justify calling $F(\mathbf{x}(t), \stackrel{\circ}{\mathbf{x}}(t))$ a generalized submetric function..$^{9}$

We show next that a smooth path always allows for a smooth parametrization.

Theorem 3 A parametrization $\mathbf{x} \mid[a, b]$ of a smooth path is smooth if and only if it is a positive diffeomorphic reparametrization of its natural $D$-parametrization. In particular, the natural $D$-parametrization of a smooth $\mathbf{x} \mid[a, b]$ is smooth.

Proof. The ratio

$$
\frac{D \mathbf{x}(t) \mathbf{x}(t+s)}{D \mathbf{x}([t, t+s])}
$$

is clearly invariant under all (continuous) reparametrizations, including the natural $D$-parametrization $\mathbf{n} \mid[0, D \mathbf{x}([a, b])]$ defined by $\mathbf{x}(x)=\mathbf{n}(\phi(x))$ with $\phi(x)=D \mathbf{x}([a, x])$. So,

$$
\frac{D \mathbf{x}(t) \mathbf{x}(t+s)}{D \mathbf{x}([t, t+s])}=\frac{D \mathbf{n}(\phi(t)) \mathbf{n}(\phi(t+s))}{D \mathbf{n}([\phi(t), \phi(t+s)])} .
$$

We have then, on denoting $\rho=\phi(t)$ and $\sigma=\phi(t+s)-\phi(t)$,

$$
1=\lim _{s \rightarrow 0+} \frac{D \mathbf{n}(\phi(t)) \mathbf{n}(\phi(t+s))}{D \mathbf{n}([\phi(t), \phi(t+s)])}=\lim _{\sigma \rightarrow 0+} \frac{D \mathbf{n}(\rho) \mathbf{n}(\rho+\sigma)}{\sigma} .
$$

This proves the second statement of the theorem, as the function

$$
\lim _{\sigma \rightarrow 0+} \frac{D \mathbf{n}(\rho) \mathbf{n}(\rho+\sigma)}{\sigma}=F_{\mathbf{n}}(\mathbf{n}(\rho), \stackrel{\circ}{\mathbf{n}}(\rho)) \equiv 1
$$

is positive and continuous.

Next we observe that

$$
\frac{D \mathbf{x}(t) \mathbf{x}(t+s)}{s}=\frac{D \mathbf{n}(\phi(t)) \mathbf{n}(\phi(t+s))}{D \mathbf{n}([\phi(t), \phi(t+s)])} \cdot \frac{\phi(t+s)-\phi(t)}{s},
$$

\footnotetext{
${ }^{9}$ See footnote 5 for the properties of a submetric function in Finsler geometry, broadly understood. The present account of $F(\mathbf{x}(t), \stackrel{\circ}{\mathbf{x}}(t))$ is simpler than in Dzhafarov and Colonius (2005a) because Property $\mathcal{D} 4$ of dissimilarity stated in Section 2.2 allows one to circumvent the theory of regular variation which played a prominent role in the previous versions of Fechnerian Scaling (see Dzhafarov 2002a-d).
} 
whence

$$
\lim _{s \rightarrow 0+} \frac{D \mathbf{x}(t) \mathbf{x}(t+s)}{s}=\lim _{s \rightarrow 0+} \frac{\phi(t+s)-\phi(t)}{s}=\frac{\mathrm{d} \phi(t)}{\mathrm{d} t+} .
$$

We see that

$$
F(\mathbf{x}(t), \stackrel{\circ}{\mathbf{x}}(t))=\lim _{s \rightarrow 0+} \frac{D \mathbf{x}(t) \mathbf{x}(t+s)}{s}
$$

exists as a positive continuous functions if and only if so does $\mathrm{d} \phi(t) / \mathrm{d} t+$. But then (see, e.g., Bruckner, 1978, p. 53)

$$
\frac{\mathrm{d} \phi(t)}{\mathrm{d} t}=\frac{\mathrm{d} \phi(t)}{\mathrm{d} t+}
$$

i.e., $\phi$ is a positive diffeomorphism.

Theorem 4 For a smooth parametrization $\mathbf{x} \mid[a, b]$ of a smooth path, as $s \rightarrow 0+$,

$$
\frac{D \mathbf{x}(t) \mathbf{x}(t+s) / s}{F(\mathbf{x}(t), \stackrel{\circ}{\mathbf{x}}(t))} \rightarrow 1
$$

uniformly.

Proof. Using the same argument and notation as in the proof of Theorem 3,

$$
\frac{D \mathbf{x}(t) \mathbf{x}(t+s) / s}{F(\mathbf{x}(t), \stackrel{\circ}{\mathbf{x}}(t))}=\frac{D \mathbf{n}(\phi(t)) \mathbf{n}(\phi(t+s))}{D \mathbf{n}([\phi(t), \phi(t+s)])} \cdot \frac{(\phi(t+s)-\phi(t)) / s}{\mathrm{~d} \phi(t) / \mathrm{d} t} .
$$

The left multiplicand tends to 1 uniformly by the definition of a smooth path. The right multiplicand is continuous on $[a, b] \times\left[0, s_{0}\right]$ (where $s_{0}$ is any positive number), so it is uniformly continuous, hence converging to 1 uniformly.

The next result can be viewed as a justification for our definition of a smooth path.

Theorem 5 For a smooth and smoothly parametrized path $\mathbf{x} \mid[a, b]$,

$$
D \mathbf{x}([a, b])=\int_{a}^{b} F(\mathbf{x}(t), \stackrel{\circ}{\mathbf{x}}(t)) \mathrm{d} t .
$$

Proof. By Theorem 1, for any sequence of nets $\mu_{n}=\left(a=t_{0}^{n}, t_{1}^{n}, \ldots, t_{k_{n}}^{n}, t_{k_{n}+1}^{n}=b\right)$ with $\delta \mu_{n} \rightarrow 0$,

$$
D \mathbf{x}([a, b])=\lim _{n \rightarrow \infty} \sum_{i=0}^{k_{n}} D \mathbf{x}\left(t_{i}^{n}\right) \mathbf{x}\left(t_{i+1}^{n}\right)=\lim _{n \rightarrow \infty} \sum_{i=0}^{k_{n}} \frac{D \mathbf{x}\left(t_{i}^{n}\right) \mathbf{x}\left(t_{i+1}^{n}\right)}{t_{i+1}^{n}-t_{i}^{n}}\left(t_{i+1}^{n}-t_{i}^{n}\right) .
$$

By Theorem 4,

$$
\frac{\frac{D \mathbf{x}\left(t_{i}^{n}\right) \mathbf{x}\left(t_{i+1}^{n}\right)}{t_{i+1}^{n}-t_{i}^{n}}\left(t_{i+1}^{n}-t_{i}^{n}\right)}{F\left(\mathbf{x}\left(t_{i}^{n}\right), \stackrel{\circ}{\mathbf{x}}\left(t_{i}^{n}\right)\right)\left(t_{i+1}^{n}-t_{i}^{n}\right)} \rightarrow 1
$$

uniformly as $\delta \mu_{n} \rightarrow 0$, whence

$$
\lim _{n \rightarrow \infty} \frac{\sum_{i=0}^{k_{n}} \frac{D \mathbf{x}\left(t_{i}^{n}\right) \mathbf{x}\left(t_{i+1}^{n}\right)}{t_{i+1}^{n}-t_{i}^{n}}\left(t_{i+1}^{n}-t_{i}^{n}\right)}{\sum_{i=0}^{k_{n}} F\left(\mathbf{x}\left(t_{i}^{n}\right), \stackrel{\circ}{\mathbf{x}}\left(t_{i}^{n}\right)\right)\left(t_{i+1}^{n}-t_{i}^{n}\right)}=1 .
$$


But, due to the continuity of $F$ in $t$ and by the definition of Riemann integral,

$$
\lim _{n \rightarrow \infty} \sum_{i=0}^{k_{n}} F\left(\mathbf{x}\left(t_{i}^{n}\right), \stackrel{\circ}{\mathbf{x}}\left(t_{i}^{n}\right)\right)\left(t_{i+1}^{n}-t_{i}^{n}\right)=\int_{a}^{b} F(\mathbf{x}(t), \stackrel{\circ}{\mathbf{x}}(t)) \mathrm{d} t .
$$

This establishes the result.

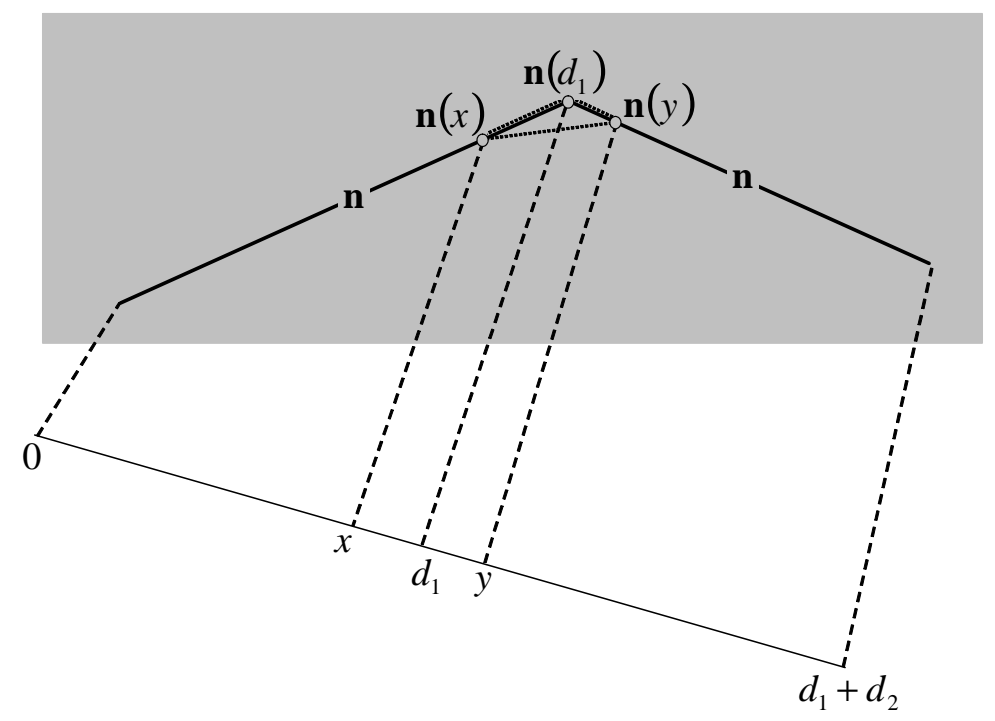

Figure 8. An example of a smoothly parametrized path which is not smooth. A path $\mathbf{n} \mid\left[0, d_{1}+d_{2}\right]$ is shown as a mapping into Euclidean plane (grey area) of an interval $\left[0, d_{1}+d_{2}\right]$, with the image consisting of two concatenated straight line segments. This path is naturally $D$-parametrized with respect to Euclidean distance taken as the dissimilarity $D$ : this means that the Euclidean length ( $=D$-length) of the path from its left endpoint to any point $\mathbf{n}(x)$ equals $x$. Clearly, the ratio of $D \mathbf{n}(x) \mathbf{n}\left(d_{1}\right)+$ $D \mathbf{n}\left(d_{1}\right) \mathbf{n}(y)$ to $D \mathbf{n}(x) \mathbf{n}(y)$ does not tend to 1 with $x$ and $y$ converging to $d_{1}$.

Theorem 3 should not be construed to indicate interchangeability between the properties of being smooth and being smoothly parametrized: neither of these two properties generally implies the other. A smooth path can be parametrized non-smoothly: to achieve this, as follows from Theorem 3, it would suffice to reparametrize a smooth parametrization by means of a non-diffeomorphic homeomorphism of its domain. A smoothly parametrized path, on the other hand, need not be smooth. For instance, a concatenation of two smooth paths can always be smoothly parametrized but generally is not smooth. The concatenation of paths $\mathbf{x} \mid[a, b]$ and $\mathbf{y} \mid[c, d]$ with $\mathbf{x}(b)=\mathbf{y}(c)$ is the path $\mathbf{z} \mid[a, b-c+d]$ defined by

$$
\mathbf{z}(t)=\left\{\begin{array}{cc}
\mathbf{x}(t) & \text { if } t<b \\
\mathbf{y}(t-b+c) & \text { if } t \geq b
\end{array} .\right.
$$

Let $\mathbf{n}_{1} \mid\left[0, d_{1}\right]$ and $\mathbf{n}_{2} \mid\left[0, d_{2}\right]$ be naturally $D$-parametrized, with $\mathbf{n}_{1}\left(d_{1}\right)=\mathbf{n}_{2}(0)$. Their concatenation $\mathbf{n} \mid\left[0, d_{1}+d_{2}\right]$ defined by $(29)$ is clearly a natural $D$-parametrization, with

$$
F(\mathbf{n}(\rho), \stackrel{\circ}{\mathbf{n}}(\rho))=\lim _{\sigma \rightarrow 0+} \frac{D \mathbf{n}(\rho) \mathbf{n}(\rho+\sigma)}{\sigma}=\left\{\begin{array}{ll}
\lim _{\sigma \rightarrow 0+} \frac{D \mathbf{n}_{1}(\rho) \mathbf{n}_{1}(\rho+\sigma)}{\sigma}=1 & \text { if } \rho<d_{1} \\
\lim _{\sigma \rightarrow 0+} \frac{D \mathbf{n}_{2}(\rho) \mathbf{n}_{2}(\rho+\sigma)}{\sigma}=1 & \text { if } \rho \geq d_{1}
\end{array} .\right.
$$


Since $F(\mathbf{n}(\rho), \stackrel{\circ}{\mathbf{n}}(\rho))=1$ on its entire domain, $\mathbf{n} \mid\left[0, d_{1}+d_{2}\right]$ is smoothly parametrized. It is easy to construct examples, however, when $\mathbf{n} \mid\left[0, d_{1}+d_{2}\right]$ violates the additivity-in-the-small property (Theorem 2) at the concatenation point, and is not therefore smooth (see Fig. 8).

\section{Spaces With Simple Bases}

Recall the definition of an arc in Section 2.3: in the following we tacitly assume that all arcs we deal with are homeomorphically parametrized. We now introduce smooth arcs of a special kind. It is convenient to denote them by indicating their initial and terminal points, as in $\mathbf{u}_{\mathbf{a}}^{\mathbf{b}}$, and omitting their specific parametrization $\mathbf{u} \mid[a, b]$ (but $\mathbf{u}_{\mathbf{a}}^{\mathbf{b}}$ does not imply that $\mathbf{u} \mid[a, b]$ is uniquely determined by its endpoints).

A set of smooth $\operatorname{arcs} \mathcal{R}$ is said to form a uniformly simple system (and its elements are referred to as simple arcs $)$ if whenever $\mathbf{u}_{\mathbf{a}_{n}}^{\mathbf{b}_{n}} \in \mathcal{R}\left(\mathbf{a}_{n} \neq \mathbf{b}_{n}\right)$ and $\mathbf{a}_{n} \leftrightarrow \mathbf{b}_{n}$,

$$
\frac{D \mathbf{u}_{\mathbf{a}_{n}}^{\mathbf{b}_{n}}}{D \mathbf{a}_{n} \mathbf{b}_{n}} \rightarrow 1
$$

In other words, we require that within a uniformly simple system of arcs the approximation of pairwise dissimilarities by lengths of simple arcs be uniform.

For instance, if $\mathbf{u} \mid[a, b]$ is a smooth arc, then the set $\mathcal{R}_{\mathbf{u}}$ of all subarcs $\mathbf{u}_{\mathbf{u}(t)}^{\mathbf{u}(\tau)}, a \leq t, \tau \leq b$, is a uniformly simple system. Indeed, $\mathbf{u}(t) \leftrightarrow \mathbf{u}(\tau)$ if and only if $\tau-t \rightarrow 0$, and

$$
\frac{D \mathbf{u}_{\mathbf{u}(t)}^{\mathbf{u}(\tau)}}{D \mathbf{u}(t) \mathbf{u}(\tau)} \rightarrow 1
$$

by the definition of a smooth path.

Clearly, any subset of a uniformly simple system is a uniformly simple system, and so is a finite union of uniformly simple systems.

Now we formulate the main definition of this section (see Fig. 9). A ball $\mathfrak{B}_{D}(\mathbf{p}, r)$ is called simple if any two points $\mathbf{x}, \mathbf{y} \in \mathfrak{B}_{D}(\mathbf{p}, r)$ are connected by an $\operatorname{arc} \mathbf{u}_{\mathbf{x}}^{\mathbf{y}}$ such that the set

$$
\mathcal{R}_{\mathbf{p}, r}=\left\{\mathbf{u}_{\mathbf{x}}^{\mathbf{y}}: \mathbf{x}, \mathbf{y} \in \mathfrak{B}_{D}(\mathbf{p}, r)\right\}
$$

forms a uniformly simple system (hence the $\operatorname{arcs} \mathbf{u}_{\mathbf{x}}^{\mathbf{y}}$ are simple arcs). A space $(\mathfrak{S}, D)$ is said to have a simple basis if for every $\mathbf{p} \in \mathfrak{S}$ there is an $r>0$ such that $\mathfrak{B}_{D}(\mathbf{p}, r)$ is a simple ball.

Clearly, if $\mathfrak{B}_{D}(\mathbf{p}, r)$ is a simple ball, then so is $\mathfrak{B}_{D}(\mathbf{p}, \varepsilon)$ for any $\varepsilon<r$. The term "space with a simple basis" reflects the obvious fact that the set of all simple balls $\mathfrak{B}_{D}(\mathbf{p}, \varepsilon)$ in a space with a simple basis forms a basis for the $D$-topology of the space.

An analogous definition can be given for a simple $G$-ball: call $\mathfrak{B}_{G}(\mathbf{p}, g)$ a simple $G$-ball if any $\mathbf{x}, \mathbf{y} \in$ $\mathfrak{B}_{G}(\mathbf{p}, g)$ are connected by an $\operatorname{arc} \mathbf{u}_{\mathbf{x}}^{\mathbf{y}}$ such that the set

$$
\mathcal{G}_{\mathbf{p}}=\left\{\mathbf{u}_{\mathbf{x}}^{\mathbf{y}}: \mathbf{x}, \mathbf{y} \in \mathfrak{B}_{G}(\mathbf{p}, g)\right\}
$$




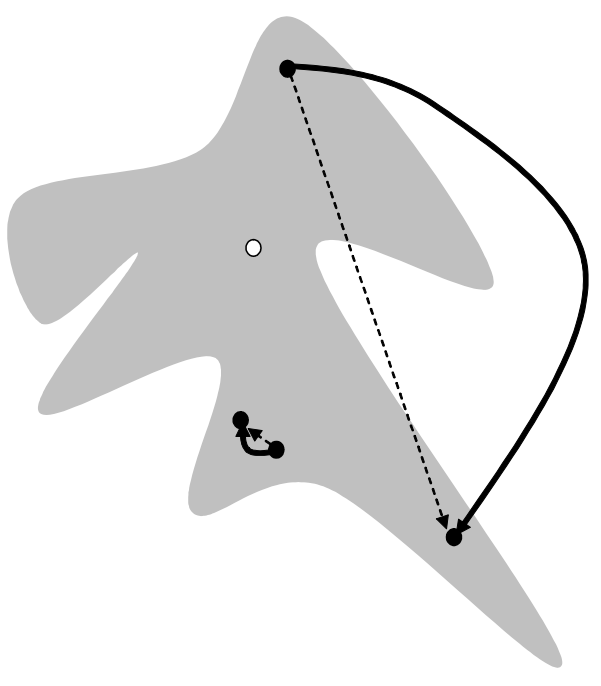

Figure 9. A schematic representation of a simple $D$-ball (or a simple $G$-ball) centered at the point shown by the open circle. Any two points within the ball can be joined by a simple arc (thick line) whose $D$-length approximates the dissimilarity between the points (dashed line): the ratio of the two converges to 1 as the dissimilarity gets smaller.

forms a uniformly simple system. ${ }^{10}$ Due to the coincidence of the $D$-topology and $G$-topology, every simple $D$-ball contains a simple $G$-ball around any of its points, and vice versa. It follows that a space has a simple basis if and only if a simple $G$-ball $\mathfrak{B}_{G}(\mathbf{p}, g)$ exists for every $\mathbf{p}$. It makes no difference therefore whether the space's topology is viewed as based on balls $\mathfrak{B}_{D}(\mathbf{p}, r)$ or on balls $\mathfrak{B}_{G}(\mathbf{p}, g)$.

Note that if $\mathbf{x}, \mathbf{y}$ lie within a simple ball (whether $D$-ball or $G$-ball), the image of a simple arc $\mathbf{u}_{\mathbf{x}}^{\mathbf{y}}$ connecting them need not lie within the ball entirely (as shown in Fig. 9).

The main result of this section is that every rectifiable path connecting $\mathbf{a}$ to $\mathbf{b}$ in a space with a simple basis can be approximated pointwise and in its $D$-length by a piecewise smooth (in fact, piecewise simple) path connecting $\mathbf{a}$ to $\mathbf{b}$. We first need two auxiliary observations.

The idea and the proof of the first of them are due to Busemann (2005, p. 33). Let us call $g(\mathbf{p})$ an extended continuous function if $g(\mathbf{p})$ is either finite (real-valued) and continuous, or $g \equiv \infty$.

Lemma 2 In a space with a simple basis, there is an extended continuous function $g(\mathbf{p})$ such that all $\mathfrak{B}_{G}(\mathbf{p}, g(\mathbf{p}))$ are simple $G$-balls.

Proof. Since a simple $G$-ball $\mathfrak{B}_{G}(\mathbf{p}, g)$ exists for every $\mathbf{p}$, the function

$$
g(\mathbf{p})=\sup \left\{g: \mathfrak{B}_{G}(\mathbf{p}, g) \text { is simple }\right\}
$$

is well defined. Clearly, $\mathfrak{B}_{G}(\mathbf{p}, g(\mathbf{p}))$ is a simple $G$-ball itself. We prove that $g(\mathbf{p})$ is an extended continuous

\footnotetext{
${ }^{10}$ To prevent confusion: the uniformly simple system here is defined in the same way as above, in terms of the ratios $D \mathbf{u}_{\mathbf{a}_{n}}^{\mathbf{b}_{n}} / D \mathbf{a}_{n} \mathbf{b}_{n} \rightarrow 1$ within the ball. $D$ is not being replaced with $G$ here, only the definition of $\mathbf{a}_{n}, \mathbf{b}_{n}$ being close to $\mathbf{p}$ changes.
} 
function. If $g=\infty$ for a single $\mathbf{p}$, then $g \equiv \infty$. Assume that $g$ is finite. We show that whenever $g(\mathbf{p})>g(\mathbf{q})$,

$$
g(\mathbf{p})-g(\mathbf{q}) \leq G \mathbf{p q} .
$$

This is obvious if $g(\mathbf{p}) \leq G \mathbf{p q}$. If $g(\mathbf{p})>G \mathbf{p q}$, then

$$
\mathfrak{B}_{G}(\mathbf{p}, g(\mathbf{p})) \supset \mathfrak{B}_{G}(\mathbf{q}, g(\mathbf{p})-G \mathbf{p q}) .
$$

Indeed, if $G \mathbf{q} \mathbf{x}<g(\mathbf{p})-G \mathbf{p q}$, then

$$
G \mathbf{p x} \leq G \mathbf{p q}+G \mathbf{q} \mathbf{x}<g(\mathbf{p})
$$

Hence $g(\mathbf{p})-G \mathbf{p q} \leq g(\mathbf{q})$.

To formulate our second observation, let $\mathbf{u}_{\mathbf{a}}^{\mathbf{b}}$ be an arc. Denote by $M \mathbf{u}_{\mathbf{a}}^{\mathbf{b}}$ the maximum of $D \mathbf{a x}$ across all $\mathbf{x}$ belonging to the image of $\mathbf{u}_{\mathbf{a}}^{\mathbf{b}}$.

Lemma 3 If $\mathbf{a}_{n} \leftrightarrow \mathbf{b}_{n}\left(\mathbf{a}_{n} \neq \mathbf{b}_{n}\right)$ within a simple ball $\mathfrak{B}_{D}(\mathbf{p}, r)$, or $\mathfrak{B}_{G}(\mathbf{p}, g)$, then $M \mathbf{u}_{\mathbf{a}_{n}}^{\mathbf{b}_{n}} \rightarrow 0$.

Proof. Assume the contrary: $M \mathbf{u}_{\mathbf{a}_{n}}^{\mathbf{b}_{n}} \nrightarrow 0$ for some sequence $\mathbf{a}_{n} \leftrightarrow \mathbf{b}_{n}$. Since the value of $M \mathbf{u}_{\mathbf{a}_{n}}^{\mathbf{b}_{n}}$ is attained by $D \mathbf{a}_{n} \mathbf{x}$ at some $\mathbf{x}_{n}$ in the image of $\mathbf{u}_{\mathbf{a}_{n}}^{\mathbf{b}_{n}}$, we have then a sequence $\left(\mathbf{a}_{n}, \mathbf{x}_{n}\right)$ with $D \mathbf{a}_{n} \mathbf{x}_{n} \not 0$. But then $D \mathbf{u}_{\mathbf{a}_{n}}^{\mathbf{x}_{n}} \nrightarrow 0$ for the subarcs $\mathbf{u}_{\mathbf{a}_{n}}^{\mathbf{x}_{n}}$ of $\mathbf{u}_{\mathbf{a}_{n}}^{\mathbf{b}_{n}}$, implying $D \mathbf{u}_{\mathbf{a}_{n}}^{\mathbf{b}_{n}} \nrightarrow 0$. The latter, however, contradicts

$$
\frac{D \mathbf{u}_{\mathbf{a}_{n}}^{\mathbf{b}_{n}}}{D \mathbf{a}_{n} \mathbf{b}_{n}} \rightarrow 1
$$

and $D \mathbf{a}_{n} \mathbf{b}_{n} \rightarrow 0$.

Now we can state the main result of this section, which is quite intuitive (Fig. 10).

Theorem 6 In a space $(\mathfrak{S}, D)$ with a simple basis, for every $D$-rectifiable path $\mathbf{x} \mid[a, b]$ connecting $\mathbf{a}$ to $\mathbf{b}$ one can find a sequence of piecewise simple (hence piecewise smooth) paths $\mathbf{u}_{n} \mid[a, b]$ connecting $\mathbf{a}$ to $\mathbf{b}$ such that

$$
\mathbf{u}_{n}|[a, b] \rightarrow \mathbf{x}|[a, b]
$$

and

$$
D \mathbf{u}_{n}([a, b]) \rightarrow D \mathbf{x}([a, b])
$$

Proof. Observe first that the function $g(\mathbf{x}(t))$ for simple $G$-balls $\mathfrak{B}_{G}(\mathbf{x}(t), g(\mathbf{x}(t)))$ is an extended continuous function on $[a, b]$ (Lemma 2), so

$$
g_{0}=\min _{t \in[a, b]} g(\mathbf{x}(t))
$$

is a positive number or $\infty$. In either case the $G$-balls $\mathfrak{B}_{G}\left(\mathbf{x}(t), g_{0}\right)$ are simple. Observe next that by appropriately choosing a net

$$
\left(a=t_{0}, t_{1}, \ldots, t_{k}, t_{k+1}=b\right)
$$




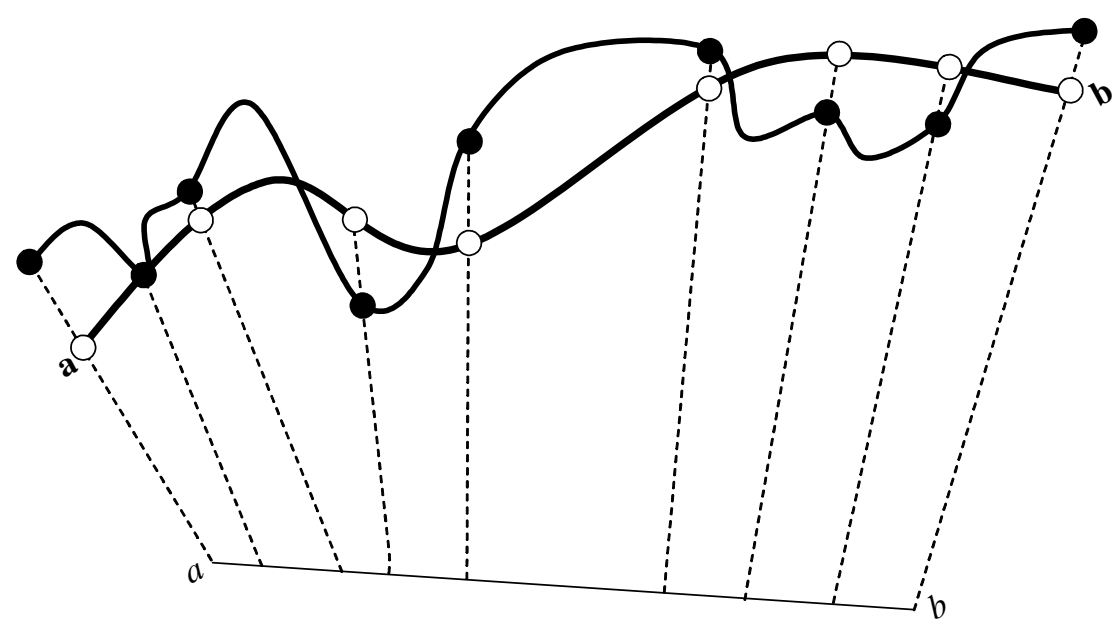

Figure 10. An illustration for Theorem 6 (compare to Fig. 3). A chain (filled circles) converging to a path and approximating its length can be replaced with a concatenation of simple arcs (curves connecting the filled circles) approximating the length of the chain and thereby the length of the path.

one can achieve

$$
\max _{t \in\left[t_{i}, t_{i+1}\right]} G \mathbf{x}\left(t_{i}\right) \mathbf{x}(t) \leq \frac{g_{0}}{2}
$$

Indeed, once $t_{i}$ is chosen for some $i$ (and for $i=0$ it is guaranteed), $G \mathbf{x}\left(t_{i}\right) \mathbf{x}(t)$ for $t \geq t_{i}$ must reach $g_{0} / 2$ for the first time at some point $t_{i+1}$, unless it never reaches it on $\left[t_{i}, b\right]$, in which case we put $t_{i+1}=b$. The sequence $t_{0}, t_{1}, \ldots$ thus formed must end in $b$ at some step, because otherwise $t_{i+1}-t_{i}$ would converge to zero as $i$ increases, and since

$$
G \mathbf{x}\left(t_{i}\right) \mathbf{x}\left(t_{i+1}\right)=g_{0} / 2
$$

by construction, this would contradict the uniform continuity of $\mathbf{x} \mid[a, b]$. With the net

$$
\left(a=t_{0}, t_{1}, \ldots, t_{k}, t_{k+1}=b\right)
$$

constructed, the set of $G$-balls $\mathfrak{B}_{G}\left(\mathbf{x}\left(t_{i}\right), g_{0}\right)(i=0, \ldots, k)$ forms a uniformly simple system covering the path $\mathbf{x} \mid[a, b]$ : specifically, each $G$-ball $\mathfrak{B}_{G}\left(\mathbf{x}\left(t_{i}\right), g_{0}\right)$ contains the subpath $\mathbf{x} \mid\left[t_{i}, t_{i+1}\right]$ with

$$
D \mathbf{x}\left(t_{i}\right) \mathbf{x}(t) \leq \frac{g_{0}}{2}
$$

for all $t \in\left[t_{i}, t_{i+1}\right]$.

Clearly, it is sufficient to prove the theorem separately for any of the fragments $\mathbf{x} \mid\left[t_{i}, t_{i+1}\right]$ contained in the simple $G$-ball $\mathfrak{B}_{G}\left(\mathbf{x}(\alpha), g_{0}\right)$, say, for

$$
\left[\alpha=t_{i}, t_{i+1}=\beta\right] .
$$

Consider a sequence of chains-on-nets

$$
\mathbf{X}_{n}^{\mu_{n}}=\left((\alpha, \mathbf{x}(\alpha))=\left(x_{0}^{n}, \mathbf{x}_{0}^{n}\right),\left(x_{1}^{n}, \mathbf{x}_{1}^{n}\right), \ldots,\left(x_{k_{n}}^{n}, \mathbf{x}_{k_{n}}^{n}\right),\left(x_{k_{n}+1}^{n}, \mathbf{x}_{k_{n}+1}^{n}\right)=(\beta, \mathbf{x}(\beta))\right)
$$


such that

$$
\mathbf{X}_{n}^{\mu_{n}} \rightarrow \mathbf{x} \mid[\alpha, \beta]
$$

and

$$
D \mathbf{X}_{n} \rightarrow D \mathbf{x}([\alpha, \beta])
$$

It is easy to see that for all sufficiently large $n$,

$$
\mathbf{x}_{0}^{n}, \mathbf{x}_{1}^{n}, \ldots, \mathbf{x}_{k_{n}}^{n}, \mathbf{x}_{k_{n}+1}^{n} \in \mathfrak{B}_{G}\left(\mathbf{x}(\alpha), g_{0}\right)
$$

Indeed, as

$$
\sigma_{\mathbf{x} \mid[\alpha, \beta]}\left(\mathbf{X}_{n}^{\mu_{n}}\right) \rightarrow 0
$$

we have both

$$
G \mathbf{x}(\alpha) \mathbf{x}\left(x_{i}^{n}\right)-G \mathbf{x}(\alpha) \mathbf{x}_{i}^{n} \rightarrow 0
$$

for all possible $x_{i}^{n}$ uniformly, and

$$
\frac{g_{0}}{2} \geq G \mathbf{x}(\alpha) \mathbf{x}\left(x_{i}^{n}\right) .
$$

It follows that each $\mathbf{x}_{i}^{n}$ is connected to $\mathbf{x}_{i+1}^{n}$ by a simple $\operatorname{arc} \mathbf{u}_{\mathbf{x}_{i}^{n}}^{\mathbf{x}_{i+1}^{n}}\left(i=0,1, \ldots, k_{n}\right)$.

Denoting the concatenation of these arcs (a piecewise simple path) by $\mathbf{u} \mid[\alpha, \beta]$, let $\left(m_{n}, \mathbf{x}\left(m_{n}\right)\right)$ be the point of $\mathbf{x} \mid[\alpha, \beta]$ at which

$$
D \mathbf{x}\left(m_{n}\right) \mathbf{u}\left(m_{n}\right)=\sigma_{\mathbf{x} \mid[\alpha, \beta]}(\mathbf{u} \mid[\alpha, \beta]) .
$$

Let $x_{i_{n}} \leq m_{n}<x_{i_{n}+1}$ for two successive elements of $\mu_{n}$. By the uniform continuity of $\mathbf{x} \mid[\alpha, \beta]$,

$$
D \mathbf{x}\left(x_{i_{n}}\right) \mathbf{x}\left(m_{n}\right) \rightarrow 0
$$

and by Lemma 3 ,

$$
D \mathbf{u}\left(x_{i_{n}}\right) \mathbf{u}\left(m_{n}\right) \rightarrow 0
$$

Combined with

$$
D \mathbf{x}\left(x_{i_{n}}\right) \mathbf{u}\left(x_{i_{n}}\right) \rightarrow 0
$$

this proves the first statement of the theorem:

$$
\sigma_{\mathbf{x} \mid[\alpha, \beta]}(\mathbf{u} \mid[\alpha, \beta])=D \mathbf{x}\left(m_{n}\right) \mathbf{u}\left(m_{n}\right) \rightarrow 0 .
$$

Now, as $\delta \mu_{n} \rightarrow 0$,

$$
\max _{i=0,1, \ldots, k_{n}} D \mathbf{x}_{i}^{n} \mathbf{x}_{i+1}^{n} \rightarrow 0,
$$

and, for any $\varepsilon>0, n$ can be chosen sufficiently large to ensure

$$
1-\varepsilon<\frac{D \mathbf{u}_{\mathbf{x}_{i+1}^{n}}^{\mathbf{x}_{n}^{n}}}{D \mathbf{x}_{i}^{n} \mathbf{x}_{i+1}^{n}}<1+\varepsilon
$$


for all $i=0, \ldots, k_{n}$. Then

$$
1-\varepsilon<\frac{\sum_{i=0}^{k_{n}} D \mathbf{u}_{\mathbf{x}_{i}^{n}}^{\mathbf{x}_{i+1}^{n}}}{\sum_{i=0}^{k_{n}} D \mathbf{x}_{i}^{n} \mathbf{x}_{i+1}^{n}}<1+\varepsilon .
$$

But, since $D \mathbf{X}_{n} \rightarrow D \mathbf{x}([\alpha, \beta])$, for any $\delta>0$ and sufficiently large $n$,

$$
1-\delta<\frac{\sum_{i=0}^{k_{n}} D \mathbf{x}_{i}^{n} \mathbf{x}_{i+1}^{n}}{D \mathbf{x}([\alpha, \beta])}<1+\delta
$$

It follows that $n$ can be chosen so large that

$$
\frac{1-\varepsilon}{1+\delta}<\frac{\sum_{i=0}^{k_{n}} D \mathbf{u}_{\mathbf{x}_{i+1}^{n}}^{\mathbf{x}_{i+1}^{n}}}{D \mathbf{x}([\alpha, \beta])}<\frac{1+\varepsilon}{1-\delta},
$$

whence the second statement of the theorem follows, on observing that the two boundaries can be made arbitrarily close to 1 .

For completeness, we mention an obvious by-product of this proof.

Theorem 7 In a space with a simple basis any two points $\mathbf{a}, \mathbf{b}$ can be connected by a piecewise smooth (in fact, piecewise simple) path. Moreover, the infimum of the $D$-lengths of all paths connecting $\mathbf{a}$ to $\mathbf{b}$ coincides with the infimum of the $D$-lengths of all piecewise simple paths connecting $\mathbf{a}$ to $\mathbf{b}$.

The significance of this corollary to Theorem 6 is that in spaces where the Fechnerian distance Gab can be found as an infimum of the $D$-lengths of all paths connecting a to $\mathbf{b}$ (e.g., in complete spaces with intermediate points, by Proposition 16), the consideration can be confined to only piecewise smooth, even piecewise simple paths.

\section{Metric in the Small Dissimilarities}

We say that a dissimilarity function $D$ is metric in the small ${ }^{11}$ if for every $\mathbf{p} \in \mathfrak{S}$ there is an $r>0$ such that whenever $\mathbf{x}_{n} \leftrightarrow \mathbf{y}_{n}\left(\mathbf{x}_{n} \neq \mathbf{y}_{n}\right)$ within $\mathfrak{B}_{D}(\mathbf{p}, r)$ (called a small D-ball, see Fig. 11),

$$
\lim _{n \rightarrow \infty} \frac{D \mathbf{x}_{n} \mathbf{y}_{n}}{G \mathbf{x}_{n} \mathbf{y}_{n}}=1 .
$$

Clearly, a metric dissimilarity is also metric in the small.

Due to the coincidence of the $D$-topology and $G$-topology, if $D$ is a metric in the small, then every $\mathbf{p} \in \mathfrak{S}$ is a center of a $G$-ball $\mathfrak{B}_{G}(\mathbf{p}, g(\mathbf{p}))$ (called a small $G$-ball) within which the convergence (33) holds. By the same argument as in Lemma $2, g(\mathbf{p})$ can be taken to be an extended continuous function.

Recall the definition of $D_{i n s} \mathbf{x}([a, b])$ in $(21)$ and Proposition 13. The theorem below generalizes this proposition: for the equality of $D \mathbf{x}([a, b])$ and $D_{\text {ins }} \mathbf{x}([a, b])$ it is sufficient for $D$ to be metric in the small.

\footnotetext{
${ }^{11}$ The word "metric" is interchangeably used as an adjective ("is metric," "metric dissimilarity") or a noun ("is a metric").
} 


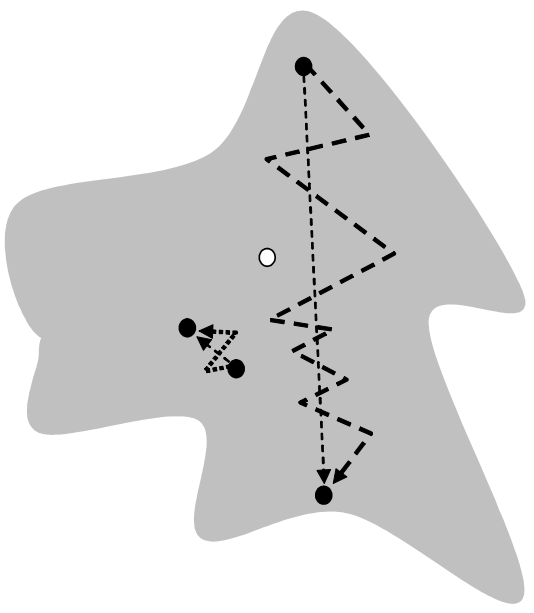

Figure 11. A schematic representation of a small $D$-ball (or a small $G$-ball) centered at the point shown by the open circle. As two points within the ball converge to each other, the infimum of the $D$-lengths for all possible chains connecting them gets progressively better approximated by the "direct" dissimilarity between them.

Theorem 8 If $D$ is a metric in the small, then

$$
D \mathbf{x}([a, b])=D_{i n s} \mathbf{x}([a, b])
$$

for any path $\mathbf{x} \mid[a, b]$.

Proof. With no loss of generality we may assume that the image $\mathbf{x}([a, b])$ of the path lies within a small ball (if it is not, we can subdivide the path into such fragments and prove the theorem for each of them). Let $\mathbf{X}_{n}^{\nu_{n}}$ be an arbitrary sequence of chains-on-nets inscribed in $\mathbf{x} \mid[a, b]$, with $\delta \nu_{n} \rightarrow 0$. The latter is equivalent to

$$
\mathbf{X}_{n}^{\nu_{n}} \rightarrow \mathbf{x} \mid[a, b]
$$

According to Proposition 14, one can construct a sequence of

$$
\mathbf{Z}_{n}^{\mu_{n}} \rightarrow \mathbf{x} \mid[a, b]
$$

with

$$
D \mathbf{Z}_{n} \rightarrow D \mathbf{x}([a, b])
$$

such that

$$
\mathbf{X}_{n}^{\nu_{n}} \subset \mathbf{Z}_{n}^{\mu_{n}} .
$$

Beginning with some $n$ we can assume (using the same argument as in Theorem 6) that all elements of $\mathbf{Z}_{n}$ lie within the small ball containing $\mathbf{x}([a, b])$.

For $i=0,1, \ldots, k_{n}$, let $\mathbf{x}\left(x_{i}^{n}\right), \mathbf{x}\left(x_{i+1}^{n}\right)$ be two successive elements of $\mathbf{X}_{n}$, and let $\mathbf{x}\left(x_{i}^{n}\right) \mathbf{Z}_{n, i} \mathbf{x}\left(x_{i+1}^{n}\right)$ be the corresponding subchain of $\mathbf{Z}_{n}$. We have then,

$$
1 \leq \frac{D \mathbf{x}\left(x_{i}^{n}\right) \mathbf{x}\left(x_{i+1}^{n}\right)}{D \mathbf{x}\left(x_{i}^{n}\right) \mathbf{Z}_{n, i} \mathbf{x}\left(x_{i+1}^{n}\right)} \leq \frac{D \mathbf{x}\left(x_{i}^{n}\right) \mathbf{x}\left(x_{i+1}^{n}\right)}{G \mathbf{x}\left(x_{i}^{n}\right) \mathbf{x}\left(x_{i+1}^{n}\right)},
$$


and as the second ratio uniformly tends to 1 as $n \rightarrow \infty$, so does the first ratio. It follows then that

$$
\lim _{n \rightarrow \infty} \frac{\sum_{i=0}^{k_{n}} D \mathbf{x}\left(x_{i}^{n}\right) \mathbf{x}\left(x_{i+1}^{n}\right)}{\sum_{i=0}^{k_{n}} D \mathbf{x}\left(x_{i}^{n}\right) \mathbf{Z}_{n, i} \mathbf{x}\left(x_{i+1}^{n}\right)}=1 .
$$

But

$$
\lim _{n \rightarrow \infty} \sum_{i=0}^{k_{n}} D \mathbf{x}\left(x_{i}^{n}\right) \mathbf{Z}_{n, i} \mathbf{x}\left(x_{i+1}^{n}\right)=D \mathbf{x}([a, b])
$$

by construction, whence

$$
\lim _{n \rightarrow \infty} \sum_{i=0}^{k_{n}} D \mathbf{x}\left(x_{i}^{n}\right) \mathbf{x}\left(x_{i+1}^{n}\right)
$$

exists and equals $D \mathbf{x}([a, b])$. Since $\mathbf{X}_{n}^{\nu_{n}}$ was chosen arbitrarily, we also have, by definition,

$$
\lim _{n \rightarrow \infty} \sum_{i=0}^{k_{n}} D \mathbf{x}\left(x_{i}^{n}\right) \mathbf{x}\left(x_{i+1}^{n}\right)=D_{i n s} \mathbf{x}([a, b]),
$$

and this proves the theorem.

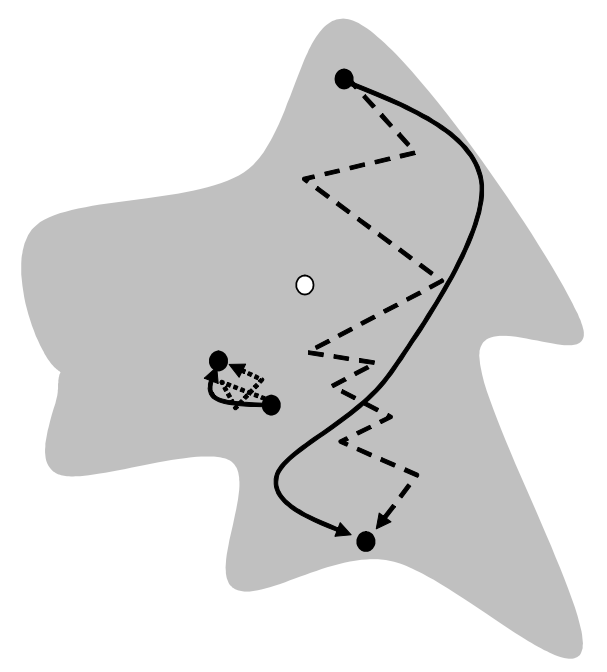

Figure 12. A schematic representation of a geodesic $D$-ball (or a geodesic $G$-ball) centered at the point shown by the open circle. As two points within the ball converge to each other, a simple arc connecting them provides progressively better approximation for the infimum of the $D$-length for all possible chains connecting them.

Let us now link the notion of a metric in the small to that of a space with a simple basis. In a space possessing both these properties the simple balls, $\mathfrak{B}_{D}(\mathbf{p}, r)$ or $\mathfrak{B}_{G}(\mathbf{p}, g)$, can always be chosen to be small, and then we call them geodesic balls. The justification for this term is as follows. Within a geodesic ball any two distinct points $\mathbf{a}_{n}, \mathbf{b}_{n}$ are connected by a simple $\operatorname{arc} \mathbf{u}_{\mathbf{a}_{n}} \mathbf{b}_{n}$, and as $\mathbf{a}_{n} \leftrightarrow \mathbf{b}_{n}$ we have both

$$
\frac{D \mathbf{u}_{\mathbf{a}_{n}}^{\mathbf{b}_{n}}}{D \mathbf{a}_{n} \mathbf{b}_{n}} \rightarrow 1
$$


because the ball is simple, and

$$
\frac{D \mathbf{a}_{n} \mathbf{b}_{n}}{G \mathbf{a}_{n} \mathbf{b}_{n}} \rightarrow 1,
$$

because the ball is small. It follows that whenever $\mathbf{a}_{n} \leftrightarrow \mathbf{b}_{n}$ within a geodesic ball,

$$
\frac{D \mathbf{u}_{\mathbf{a}_{n}}}{G \mathbf{a}_{n} \mathbf{b}_{n}} \rightarrow 1,
$$

that is, $\mathbf{u}_{\mathbf{a}_{n}}^{\mathbf{b}_{n}}$ acts as a geodesic arc in the small (see Fig. 12).

Since the geodesic balls form a basis for the $D$-topology, we can call a space with a simple basis in which $D$ is a metric in the small a space with a geodesic basis.

\section{Euclidean $n$-spaces}

We are now prepared to see how the general theory of path length (Dzhafarov, 2007), with the aid of the intermediate constructions of the previous sections, can be specialized to mathematical foundations of Multidimensional Fechnerian Scaling (MDFS) proposed in Dzhafarov and Colonius (2001) and Dzhafarov (2002a-d).

Let stimuli be represented by points in an open connected region of the Euclidean $n$-space, denoted $\mathfrak{E}$ and endowed with the usual Euclidean metric

$$
E \mathbf{a b}=|\mathbf{a}-\mathbf{b}| .
$$

Recall that the connectedness of $\mathfrak{E}$ means that it cannot be presented as a union of two open sets. In the Euclidean space this notion is equivalent to arc-connectedness: any two points can be connected by an arc.

A straight line segment is defined to be an arc parametrizable as

$$
\mathbf{s}(t)=\mathbf{a}+\mathbf{u} t, \quad t \in[a, b] .
$$

The tangent space $\mathfrak{T}_{\mathbf{p}}$ associated with every point $\mathbf{p}$ of $\mathfrak{E}$ is simply the set $\mathbb{R}^{n}$ of all $n$-vectors (directions) $\mathbf{u}$, endowed with the Euclidean norm $|\mathbf{u}|$. For any $\mathbf{u} \neq \mathbf{0}$, the notation $\overline{\mathbf{u}}$ will be used for the unit vector codirectional with $\mathbf{u}$ :

$$
\overline{\mathbf{u}}=\frac{\mathbf{u}}{|\mathbf{u}|}, \quad|\overline{\mathbf{u}}|=1 .
$$

The set of all unit vectors $\overline{\mathbf{u}}$ is denoted $\overline{\mathfrak{u}}$ (the notation is consistent with that for unit vectors and with the fact that $\overline{\mathfrak{u}}$ is closed in $\mathbb{R}^{n}$ ).

As any metric, Euclidean metric $E$ is a dissimilarity function. Straight line segments $\mathbf{a}+\mathbf{u} t$ and, generally, all $C^{1}$ (continuously differentiable) paths in $\mathfrak{E}$ are $E$-smooth and smoothly $E$-parametrized.

Let now a dissimilarity function $D$ be imposed on $\mathfrak{E}$ (e.g., $\Psi^{(1)}$ or $\Psi^{(2)}$, as discussed in the next section).

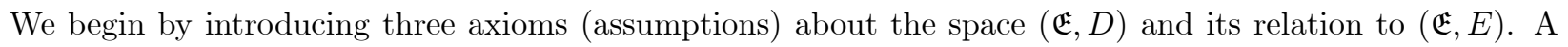
fourth axiom needed for a complete theory will be formulated after we have developed a differential geometry of path length. 
AxIOm 1 The topologies of $(\mathfrak{E}, D)$ and $(\mathfrak{E}, E)$ coincide.

The coincidence of the $D$-topology and the Euclidean topology means, of course,

$$
\mathbf{a}_{n} \leftrightarrow \mathbf{a} \Longleftrightarrow \mathbf{a}_{n} \rightarrow \mathbf{a},
$$

where $\rightarrow$ denotes the usual Euclidean convergence. In all topological considerations therefore the balls $\mathfrak{B}_{D}(\mathbf{p}, \varepsilon), \mathfrak{B}_{G}(\mathbf{p}, \varepsilon)$, and $\mathfrak{B}_{E}(\mathbf{p}, \varepsilon)$ can be used interchangeably. As a result, all topological concepts (openness, continuity, compactness, etc.) can be used without the prefix $D, G$, or $E$. In particular, dissimilarity $D \mathbf{x y}$ and metric $G \mathbf{x y}$ are continuous in $(\mathbf{x}, \mathbf{y})$ with respect to the (product) Euclidean topology.

Note, however, that the $D$-uniformity is not assumed to coincide with the Euclidean uniformity. Thus, it is possible that $D \mathbf{a}_{n} \mathbf{b}_{n} \rightarrow 0$ but $\left|\mathbf{a}_{n}-\mathbf{b}_{n}\right| \not \rightarrow 0$, or vice versa. In particular, dissimilarity $D \mathbf{x y}$ and metric $G$ xy are not generally uniformly continuous in the Euclidean sense.

Axiom 2 For any $\mathbf{p}, \mathbf{a}_{n}, \mathbf{b}_{n} \in \mathfrak{E}\left(\mathbf{a}_{n} \neq \mathbf{b}_{n}\right)$, if $\mathbf{a}_{n} \leftrightarrow \mathbf{p}$ and $\mathbf{b}_{n} \leftrightarrow \mathbf{p}$,

$$
\frac{D \mathbf{a}_{n} \mathbf{b}_{n}}{G \mathbf{a}_{n} \mathbf{b}_{n}} \rightarrow 1
$$

This axiom will determine the variant of the MDFS theory we are constructing: MDFS with convex submetric functions (equivalently, convex indicatrices). As the next theorem tells us, this axiom means that the dissimilarity $D$ is metric in the small.

Theorem 9 For any set $\mathfrak{e}$ contained in a compact subset of $\mathfrak{E}$,

$$
\frac{D \mathbf{a}_{n} \mathbf{b}_{n}}{G \mathbf{a}_{n} \mathbf{b}_{n}} \rightarrow 1
$$

as $\mathbf{a}_{n} \leftrightarrow \mathbf{b}_{n}\left(\mathbf{a}_{n} \neq \mathbf{b}_{n}\right)$ within $\mathfrak{e}$. In particular, this is true for any Euclidean ball $\mathfrak{B}_{E}(\mathbf{p}, r),{ }^{12}$ implying that $D$ is a metric in the small, and every Euclidean ball is small.

Proof. (Essentially the same as for Lemma 1.) Assume the contrary: for some sequence $\mathbf{a}_{n} \leftrightarrow \mathbf{b}_{n}$ within $\mathfrak{e}$,

$$
\frac{D \mathbf{a}_{n} \mathbf{b}_{n}}{G \mathbf{a}_{n} \mathbf{b}_{n}} \nrightarrow 1
$$

Then either lim inf or $\lim \sup$ of the ratio equals $L \neq 1$, and there is a subsequence of $\left(\mathbf{a}_{n}, \mathbf{b}_{n}\right)$ (without loss of generality, the sequence itself) for which

$$
\frac{D \mathbf{a}_{n} \mathbf{b}_{n}}{G \mathbf{a}_{n} \mathbf{b}_{n}} \rightarrow L
$$

Since $\mathfrak{e}$ is within a compact set, for some subsequence of $\left(\mathbf{a}_{n}, \mathbf{b}_{n}\right)$ and a point $\mathbf{p}$,

$$
\mathbf{a}_{n} \leftrightarrow \mathbf{p}, \mathbf{b}_{n} \leftrightarrow \mathbf{p},
$$

\footnotetext{
${ }^{12}$ This is the reason we define $\mathfrak{e}$ as contained within a compact set rather than simply compact (which would otherwise make no difference as the proof only uses the fact that $\mathbf{a}_{n}, \mathbf{b}_{n}$ vary within a compact set). Open balls $\mathfrak{B}_{E}(\mathbf{p}, r)$ are more directly related to the general definitions in Section 5 than their compact closures $\overline{\mathfrak{B}}_{E}(\mathbf{p}, r)$.
} 
and we arrive at a contradiction with Axiom 2.

The property of being metric in the small for $D$ can, of course, also be formulated in terms of $\mathfrak{B}_{D}(\mathbf{p}, r)$ or $\mathfrak{B}_{G}(\mathbf{p}, r)$ (because, by Axiom 1, every Euclidean ball contains a $D$-ball and a $G$-ball around any of its points). Note however that it need not be true that every $D$-ball or every $G$-ball is small (because they need not be contained within a Euclidean ball).

For the next axiom refer to the definition (36) of a unit vector and Fig. 13.

Axiom 3 For any $\mathbf{x}, \mathbf{a}_{n}, \mathbf{b}_{n} \in \mathfrak{E}\left(\mathbf{a}_{n} \neq \mathbf{b}_{n}\right)$ and any unit vector $\overline{\mathbf{u}}$, if $\mathbf{a}_{n} \leftrightarrow \mathbf{x}, \mathbf{b}_{n} \leftrightarrow \mathbf{x}$, and $\overline{\mathbf{b}_{n}-\mathbf{a}_{n}} \rightarrow \overline{\mathbf{u}}$ then

$$
\frac{D \mathbf{a}_{n} \mathbf{b}_{n}}{\left|\mathbf{b}_{n}-\mathbf{a}_{n}\right|}
$$

tends to a positive limit, denoted $F(\mathbf{x}, \overline{\mathbf{u}})$.

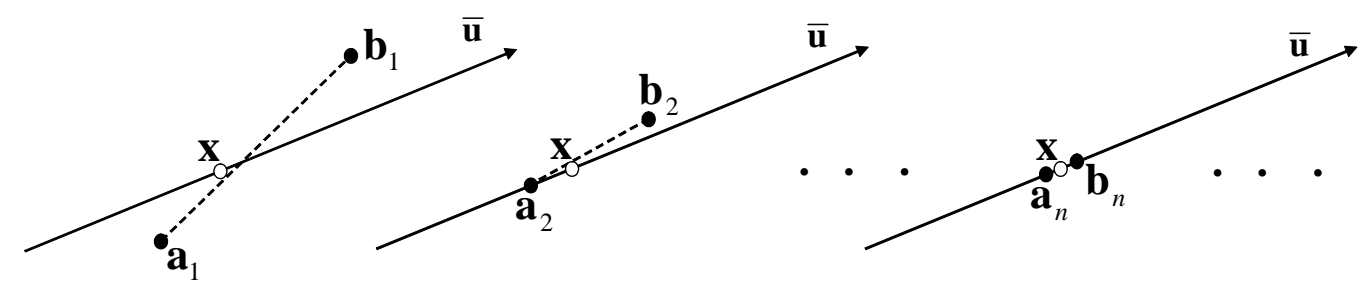

Figure 13. An illustration for Axiom 3. Shown are a point $\mathbf{x}$ (open circle), a direction $\overline{\mathbf{u}}$ attached to it, and (in successive panels from left to right) pairs of points $\left(\mathbf{a}_{1}, \mathbf{b}_{1}\right),\left(\mathbf{a}_{2}, \mathbf{b}_{2}\right), \ldots,\left(\mathbf{a}_{n}, \mathbf{b}_{n}\right), \ldots$ gradually converging to $\mathbf{x}$ so that the dashed line connecting them (and oriented from $\mathbf{a}_{n}$ to $\mathbf{b}_{n}$ ) gradually aligns with the the direction $\overline{\mathbf{u}}$. The axiom says that in this situation the dissimilarity $D \mathbf{a}_{n} \mathbf{b}_{n}$ and the Euclidean distance $\left|\mathbf{b}_{n}-\mathbf{a}_{n}\right|$ are comeasurable in the small: neither of them tends to zero infinitely faster than the other.

TheOrem $10 F(\mathbf{x}, \overline{\mathbf{u}})$ is continuous in $(\mathbf{x}, \overline{\mathbf{u}})$.

Proof. Let $\delta(\mathbf{x}, \overline{\mathbf{u}}, \varepsilon)$ (for $\varepsilon>0$ ) denote a positive quantity such that

$$
\max \{|\mathbf{a}-\mathbf{x}|,|\mathbf{b}-\mathbf{x}|,|\overline{\mathbf{b}-\mathbf{a}}-\overline{\mathbf{u}}|\}<\delta(\mathbf{x}, \overline{\mathbf{u}}, \varepsilon) \Longrightarrow\left|\frac{D \mathbf{a b}}{|\mathbf{b}-\mathbf{a}|}-F(\mathbf{x}, \overline{\mathbf{u}})\right|<\varepsilon
$$

Consider a sequence $\left(\mathbf{x}_{n}, \overline{\mathbf{u}}_{n}\right) \rightarrow(\mathbf{x}, \overline{\mathbf{u}})$, and let $\left(\mathbf{a}_{n}, \mathbf{b}_{n}\right), \mathbf{a}_{n} \neq \mathbf{b}_{n}$, be any sequence satisfying

$$
\max \left\{\left|\mathbf{a}_{n}-\mathbf{x}_{n}\right|,\left|\mathbf{b}_{n}-\mathbf{x}_{n}\right|,\left|\overline{\mathbf{b}_{n}-\mathbf{a}_{n}}-\overline{\mathbf{u}}_{n}\right|\right\}<\min \left\{\delta\left(\mathbf{x}_{n}, \overline{\mathbf{u}}_{n}, \frac{1}{n}\right), \frac{1}{2} \delta(\mathbf{x}, \overline{\mathbf{u}}, \varepsilon)\right\} .
$$

Clearly,

$$
\frac{D \mathbf{a}_{n} \mathbf{b}_{n}}{\left|\mathbf{b}_{n}-\mathbf{a}_{n}\right|}-F\left(\mathbf{x}_{n}, \overline{\mathbf{u}}_{n}\right) \rightarrow 0
$$

At the same time, for all sufficiently large $n$,

$$
\max \left\{\left|\mathbf{x}_{n}-\mathbf{x}\right|,\left|\overline{\mathbf{u}}_{n}-\overline{\mathbf{u}}\right|\right\}<\frac{1}{2} \delta(\mathbf{x}, \overline{\mathbf{u}}, \varepsilon)
$$


implying

$$
\max \left\{\left|\mathbf{a}_{n}-\mathbf{x}\right|,\left|\mathbf{b}_{n}-\mathbf{x}\right|,\left|\overline{\mathbf{b}_{n}-\mathbf{a}_{n}}-\overline{\mathbf{u}}_{n}\right|\right\}<\delta(\mathbf{x}, \overline{\mathbf{u}}, \varepsilon) .
$$

But then

$$
\left|\frac{D \mathbf{a}_{n} \mathbf{b}_{n}}{\left|\mathbf{b}_{n}-\mathbf{a}_{n}\right|}-F(\mathbf{x}, \overline{\mathbf{u}})\right|<\varepsilon,
$$

and as $\varepsilon$ can be chosen arbitrarily small, we have

$$
\frac{D \mathbf{a}_{n} \mathbf{b}_{n}}{\left|\mathbf{b}_{n}-\mathbf{a}_{n}\right|}-F(\mathbf{x}, \overline{\mathbf{u}}) \rightarrow 0 .
$$

The convergence

$$
F\left(\mathbf{x}_{n}, \overline{\mathbf{u}}_{n}\right) \rightarrow F(\mathbf{x}, \overline{\mathbf{u}})
$$

follows.

Putting $\mathbf{a}_{n}=\mathbf{x}$ and $\overline{\mathbf{b}_{n}-\mathbf{a}_{n}}=\overline{\mathbf{u}}$ in Axiom 3, and denoting $\mathbf{b}_{n}=\mathbf{x}+\overline{\mathbf{u}} s$, the function $F(\mathbf{x}, \overline{\mathbf{u}})$ can be presented as

$$
F(\mathbf{x}, \overline{\mathbf{u}})=\lim _{s \rightarrow 0+} \frac{D \mathbf{x}[\mathbf{x}+\overline{\mathbf{u}} s]}{s} .
$$

More generally, we denote

$$
F(\mathbf{x}, \mathbf{u})=\left\{\begin{array}{ll}
\lim _{s \rightarrow 0+} \frac{D \mathbf{x}[\mathbf{x}+\mathbf{u} s]}{s} & \text { if } \mathbf{u} \neq \mathbf{0} \\
0 & \text { if } \mathbf{u}=\mathbf{0}
\end{array},\right.
$$

and call $F(\mathbf{x}, \mathbf{u})$ a submetric function. We will see below (Theorem 16) that when $\mathbf{u}$ is a tangent $\dot{\mathbf{x}}(t)$ of a continuously differentiable path, $F(\mathbf{x}, \dot{\mathbf{x}}(t))$ coincides with the generalized submetric function $F(\mathbf{x}(t), \stackrel{\circ}{\mathbf{x}}(t))$ introduced in Section 3. A pair $(\mathbf{x}, \mathbf{u})$ is traditionally called a line element, and when taken in the same context it plays the role of the arc element of Section 3.

We list the standard properties of the submetric function (mentioned earlier in footnote 5).

Theorem $11 F(\mathbf{x}, \mathbf{u})$ exists for any $(\mathbf{x}, \mathbf{u}) \in \mathfrak{E} \times \mathbb{R}^{n}$. It is positive for $\mathbf{u} \neq \mathbf{0}$, continuous in $(\mathbf{x}, \mathbf{u})$, and for any $k>0, F(\mathbf{x}, k \mathbf{u})=k F(\mathbf{x}, \mathbf{u})$.

Proof. For $\mathbf{u} \neq \mathbf{0}$, denoting $\mathbf{u}=|\mathbf{u}| \overline{\mathbf{u}}$,

$$
F(\mathbf{x}, k \mathbf{u})=\lim _{s \rightarrow 0+} \frac{D \mathbf{x}[\mathbf{x}+k \mathbf{u} s]}{s}=k|\mathbf{u}| \lim _{k|\mathbf{u}| s \rightarrow 0+} \frac{D \mathbf{x}[\mathbf{x}+\overline{\mathbf{u}} k|\mathbf{u}| s]}{k|\mathbf{u}| s}=k|\mathbf{u}| F(\mathbf{x}, \overline{\mathbf{u}}) .
$$

Putting $k=1$, it immediately follows that $F(\mathbf{x}, \mathbf{u})$ exists, that it is positive and continuous, and that

$$
F(\mathbf{x}, \mathbf{u})=|\mathbf{u}| F(\mathbf{x}, \overline{\mathbf{u}}) .
$$

So

$$
F(\mathbf{x}, k \mathbf{u})=k|\mathbf{u}| F(\mathbf{x}, \overline{\mathbf{u}})=k F(\mathbf{x}, \mathbf{u}) .
$$

Finally, since any convergence of $\left(\mathbf{x}_{n}, \mathbf{u}_{n}\right) \rightarrow(\mathbf{x}, \mathbf{0})$ with $\mathbf{u}_{n} \neq \mathbf{0}$ can be presented as $\left(\mathbf{x}_{n},\left|\mathbf{u}_{n}\right| \overline{\mathbf{u}}_{n}\right) \rightarrow(\mathbf{x}, \mathbf{0})$ with $\left|\mathbf{u}_{n}\right| \rightarrow 0$, the function $F(\mathbf{x}, \mathbf{u})$ extends to $F(\mathbf{x}, \mathbf{0})=0$ by continuity.

Axiom 3 can now be strengthened as follows. 
Theorem 12 For any $\mathbf{a}_{n}, \mathbf{b}_{n} \in \mathfrak{e} \subset \mathfrak{E}$, if $\mathfrak{e}$ is compact and $\mathbf{a}_{n} \leftrightarrow \mathbf{b}_{n}\left(\mathbf{a}_{n} \neq \mathbf{b}_{n}\right)$ then

$$
\frac{D \mathbf{a}_{n} \mathbf{b}_{n}}{F\left(\mathbf{a}_{n}, \mathbf{b}_{n}-\mathbf{a}_{n}\right)} \rightarrow 1
$$

and

$$
\frac{G \mathbf{a}_{n} \mathbf{b}_{n}}{F\left(\mathbf{a}_{n}, \mathbf{b}_{n}-\mathbf{a}_{n}\right)} \rightarrow 1
$$

Proof. That the two convergences are equivalent follows from Theorem 9. It suffices to prove the first of them. Rewrite it as

$$
\frac{D \mathbf{a}_{n} \mathbf{b}_{n}}{F\left(\mathbf{a}_{n}, \overline{\mathbf{b}_{n}-\mathbf{a}_{n}}\right)\left|\mathbf{b}_{n}-\mathbf{a}_{n}\right|} \rightarrow 1
$$

If $\mathbf{a}_{n} \leftrightarrow \mathbf{x}, \mathbf{b}_{n} \leftrightarrow \mathbf{x}$, and $\overline{\mathbf{b}_{n}-\mathbf{a}_{n}} \rightarrow \overline{\mathbf{u}}$ for some line element $(\mathbf{x}, \overline{\mathbf{u}})$, then the convergence holds by Axiom 3. But within a compact set $\mathfrak{e}$ one can always select a subsequence with $\mathbf{a}_{n} \leftrightarrow \mathbf{x}, \mathbf{b}_{n} \leftrightarrow \mathbf{x}$, for some $\mathbf{x}$, from any infinite sequence $\left(\mathbf{a}_{n}, \mathbf{b}_{n}\right)$; and due to the compactness of the set $\overline{\mathfrak{u}}$ of all unit directions, one can always select a subsequence of this subsequence with $\overline{\mathbf{b}_{n}-\mathbf{a}_{n}} \rightarrow \overline{\mathbf{u}}$, for some $\overline{\mathbf{u}}$. The result now obtains using the same argument as in Theorem 9.

Submetric function $F(\mathbf{a}, \mathbf{u})$ is called convex if for any $\mathbf{a}, \mathbf{u}_{1}, \mathbf{u}_{2}$,

$$
F\left(\mathbf{a}, \mathbf{u}_{1}+\mathbf{u}_{2}\right) \leq F\left(\mathbf{a}, \mathbf{u}_{1}\right)+F\left(\mathbf{a}, \mathbf{u}_{2}\right)
$$

$F(\mathbf{a}, \mathbf{u})$ is convex if and only if the associated indicatrix at any point $\mathbf{a}$,

$$
\mathfrak{I}_{\mathbf{a}}=\{\mathbf{x}: F(\mathbf{a}, \mathbf{x}-\mathbf{a}) \leq 1\}
$$

is convex in the standard geometric meaning: if $\mathbf{a}, \mathbf{b} \in \mathfrak{I}_{\mathbf{a}}$, then the image of the straight line segment $\mathbf{a}+(\mathbf{b}-\mathbf{a}) t, t \in[0,1]$, lies entirely within $\mathfrak{I}_{\mathbf{a}}$. Note that $\mathfrak{I}_{\mathbf{a}}$ is part of the tangent space $\mathfrak{T}_{\mathbf{a}}$ rather than of $\mathfrak{G}$. For a detailed discussion of indicatrices see Dzhafarov and Colonius (2001).

Theorem 13 Submetric function $F$ is convex.

Proof. From Theorem 12 we know that for any $\mathbf{u} \neq \mathbf{0}$, if $\mathbf{a}_{n} \leftrightarrow \mathbf{a}$ and $s_{n} \rightarrow 0+$,

$$
\frac{G \mathbf{a}_{n}\left[\mathbf{a}_{n}+\mathbf{u} s_{n}\right] / s_{n}}{F\left(\mathbf{a}_{n}, \mathbf{u}\right)}=\frac{G \mathbf{a}_{n}\left[\mathbf{a}_{n}+\mathbf{u} s_{n}\right]}{F\left(\mathbf{a}_{n}, \mathbf{u} s_{n}\right)} \rightarrow 1 .
$$

Since

$$
\frac{F\left(\mathbf{a}_{n}, \mathbf{u}\right)}{F(\mathbf{a}, \mathbf{u})} \rightarrow 1
$$

we have

$$
\frac{G \mathbf{a}_{n}\left[\mathbf{a}_{n}+\mathbf{u} s_{n}\right] / s_{n}}{F(\mathbf{a}, \mathbf{u})} \rightarrow 1 .
$$

It follows that for any nonzero $\mathbf{u}_{1}, \mathbf{u}_{2}, \mathbf{u}_{1}+\mathbf{u}_{2}$,

$$
\lim _{s \rightarrow 0+} \frac{G \mathbf{a}\left[\mathbf{a}+\mathbf{u}_{1} s\right]+G\left[\mathbf{a}+\mathbf{u}_{1} s\right]\left[\left(\mathbf{a}+\mathbf{u}_{1} s\right)+\mathbf{u}_{2} s\right]}{G \mathbf{a}\left[\mathbf{a}+\left(\mathbf{u}_{1}+\mathbf{u}_{2}\right) s\right]}=\frac{F\left(\mathbf{a}, \mathbf{u}_{1}\right)+F\left(\mathbf{a}, \mathbf{u}_{2}\right)}{F\left(\mathbf{a}, \mathbf{u}_{1}+\mathbf{u}_{2}\right)} .
$$


But the left-hand expression is always $\geq 1$, whence

$$
F\left(\mathbf{a}, \mathbf{u}_{1}+\mathbf{u}_{2}\right) \leq F\left(\mathbf{a}, \mathbf{u}_{1}\right)+F\left(\mathbf{a}, \mathbf{u}_{2}\right)
$$

It remains to observe that the inequality is also valid for the cases $\mathbf{u}_{1}=\mathbf{0}, \mathbf{u}_{2}=\mathbf{0}$, and $\mathbf{u}_{1}+\mathbf{u}_{2}=\mathbf{0}$.

The next two theorems establish a special role played in the development by straight line segments.

Theorem 14 Straight line segments are smooth, and the representation $\mathbf{s}(t)=\mathbf{a}+\mathbf{u} t, t \in[a, b]$, for a straight line segment is a smooth $D$-parametrization. The $D$-length of $\mathbf{s} \mid[a, b]$ is

$$
D \mathbf{s}([a, b])=\int_{a}^{b} F(\mathbf{a}+\mathbf{u} t, \mathbf{u}) \mathrm{d} t .
$$

Proof. Since $D$ is a metric in the small, by Theorem 8 ,

$$
D \mathbf{s}([a, b])=D_{i n s} \mathbf{s}([a, b])=\lim _{n \rightarrow \infty} \sum_{i=0}^{k_{n}} D \mathbf{s}\left(t_{i}^{n}\right) \mathbf{s}\left(t_{i+1}^{n}\right)
$$

for any sequence of nets $\mu_{n}=\left\{\ldots, t_{i}^{n}, t_{i+1}^{n} \ldots\right\}$ partitioning $[a, b]$ with $\delta \mu_{n} \rightarrow 0$. Rewriting the sum as

$$
\sum_{i=0}^{k_{n}} D\left[\mathbf{a}+\mathbf{u} t_{i}^{n}\right]\left[\mathbf{a}+\mathbf{u} t_{i+1}^{n}\right]=\sum_{i=0}^{k_{n}} F\left(\mathbf{a}+\mathbf{u} t_{i}^{n}, \mathbf{u}\left(t_{i+1}^{n}-t_{i}^{n}\right)\right) \frac{D\left[\mathbf{a}+\mathbf{u} t_{i}^{n}\right]\left[\mathbf{a}+\mathbf{u} t_{i+1}^{n}\right]}{F\left(\mathbf{a}+\mathbf{u} t_{i}^{n}, \mathbf{u}\left(t_{i+1}^{n}-t_{i}^{n}\right)\right)},
$$

we observe that, by Theorem 12 , the ratios tend to 1 uniformly on the compact set $\mathbf{s}([a, b])$. Hence

$$
D \mathbf{s}([a, b])=\lim _{n \rightarrow \infty} \sum_{i=0}^{k_{n}} F\left(\mathbf{a}+\mathbf{u} t_{i}^{n}, \mathbf{u}\left(t_{i+1}^{n}-t_{i}^{n}\right)\right)=\lim _{n \rightarrow \infty} \sum_{i=0}^{k_{n}} F\left(\mathbf{a}+\mathbf{u} t_{i}^{n}, \mathbf{u}\right)\left(t_{i+1}^{n}-t_{i}^{n}\right) .
$$

Using the continuity of $F$ and the definition of Riemann integral we establish

$$
D \mathbf{s}([a, b])=\int_{a}^{b} F(\mathbf{a}+\mathbf{u} t, \mathbf{u}) \mathrm{d} t .
$$

Using (40),

$$
F(\mathbf{a}+\mathbf{u} t, \mathbf{u})=\lim _{s \rightarrow 0+} \frac{D[\mathbf{a}+\mathbf{u} t][\mathbf{a}+\mathbf{u} t+\mathbf{u} s]}{s}=\lim _{s \rightarrow 0+} \frac{D \mathbf{s}(t) \mathbf{s}(t+s)}{s},
$$

and since $F(\mathbf{a}+\mathbf{u} t, \mathbf{u})$ is positive and continuous in $t, \mathbf{s} \mid[a, b]$ is smoothly $D$-parametrized. Finally,

$$
\lim _{t-\tau \rightarrow 0+} \frac{D \mathbf{s}(t) \mathbf{s}(\tau)}{\int_{t}^{\tau} F(\mathbf{a}+\mathbf{u} x, \mathbf{u}) \mathrm{d} x}=\lim _{t-\tau \rightarrow 0+} \frac{\frac{1}{t-\tau} D \mathbf{s}(t) \mathbf{s}(\tau)}{\frac{1}{t-\tau} \int_{t}^{\tau} F(\mathbf{a}+\mathbf{u} x, \mathbf{u}) \mathrm{d} x}=1,
$$

because the limit equals

$$
\frac{F(\mathbf{a}+\mathbf{u} c, \mathbf{u})}{F(\mathbf{a}+\mathbf{u} c, \mathbf{u})}=1
$$

whenever $t \rightarrow c, \tau \rightarrow c$ for some $c \in[a, b]$, and $[a, b]$ is compact (see Lemma 1 ).

By relating this result to Theorems 9 and 12, we see that the theory we are constructing is a special case of that for spaces with geodesic bases (Section 5). 
Theorem $15(\mathfrak{E}, D)$ is a space with a geodesic basis, with straight line segments being simple arcs, and any Euclidean ball being geodesic.

Proof. By Theorem 9 , any $\mathfrak{B}_{E}(\mathbf{x}, r)$ is a small ball. To prove that it is also simple, observe that any two of its points $\mathbf{a}, \mathbf{b}$ can be connected by a straight line segment $\mathbf{s}_{\mathbf{a}}^{\mathbf{b}}$, about which we know that it is smooth (Theorem 14). We only need to show that the straight line segments in $\mathfrak{B}_{E}(\mathbf{x}, r)$ form a uniformly simple system: for any $\mathbf{a}_{n} \leftrightarrow \mathbf{b}_{n}\left(\mathbf{a}_{n} \neq \mathbf{b}_{n}\right)$,

$$
\frac{D \mathbf{a}_{n} \mathbf{b}_{n}}{D \mathbf{s}_{\mathbf{a}_{n}}^{\mathbf{b}_{n}}} \rightarrow 1
$$

Using the mean value theorem,

$$
\frac{D \mathbf{a}_{n} \mathbf{b}_{n}}{D \mathbf{s}_{\mathbf{a}_{n}}}=\frac{D \mathbf{a}_{n} \mathbf{b}_{n}}{\int_{0}^{1} F\left(\mathbf{a}_{n}+\left(\mathbf{b}_{n}-\mathbf{a}_{n}\right) x, \mathbf{b}_{n}-\mathbf{a}_{n}\right) \mathrm{d} x}=\frac{D \mathbf{a}_{n} \mathbf{b}_{n}}{F\left(\mathbf{a}_{n}+\left(\mathbf{b}_{n}-\mathbf{a}_{n}\right) \xi_{n}, \mathbf{b}_{n}-\mathbf{a}_{n}\right)}
$$

$\left(0 \leq \xi_{n} \leq 1\right)$. But

$$
\frac{D \mathbf{a}_{n} \mathbf{b}_{n}}{F\left(\mathbf{a}_{n}, \mathbf{b}_{n}-\mathbf{a}_{n}\right)} \rightarrow 1
$$

by Theorem 12, and

$$
\frac{F\left(\mathbf{a}_{n}+\left(\mathbf{b}_{n}-\mathbf{a}_{n}\right) \xi_{n}, \mathbf{b}_{n}-\mathbf{a}_{n}\right)}{F\left(\mathbf{a}_{n}, \mathbf{b}_{n}-\mathbf{a}_{n}\right)}=\frac{F\left(\mathbf{a}_{n}+\left(\mathbf{b}_{n}-\mathbf{a}_{n}\right) \xi_{n}, \overline{\mathbf{b}_{n}-\mathbf{a}_{n}}\right)}{F\left(\mathbf{a}_{n}, \overline{\mathbf{b}_{n}-\mathbf{a}_{n}}\right)} \rightarrow 1,
$$

by an argument analogous to that in Theorems 9 and 12: assuming the contrary we could choose a subsequence with $\mathbf{a}_{n} \leftrightarrow \mathbf{p}, \mathbf{b}_{n} \leftrightarrow \mathbf{p}, \overline{\mathbf{b}_{n}-\mathbf{a}_{n}} \rightarrow \overline{\mathbf{u}}$ for which the ratio would not tend to 1 , which is impossible due to

$$
\begin{aligned}
& F\left(\mathbf{a}_{n}+\left(\mathbf{b}_{n}-\mathbf{a}_{n}\right) \xi_{n}, \overline{\mathbf{b}_{n}-\mathbf{a}_{n}}\right) \rightarrow F(\mathbf{p}, \overline{\mathbf{u}}) \\
& F\left(\mathbf{a}_{n}, \overline{\mathbf{b}_{n}-\mathbf{a}_{n}}\right) \rightarrow F(\mathbf{p}, \overline{\mathbf{u}}) .
\end{aligned}
$$

This completes the proof.

A comment analogous to the one following Theorem 9 can be made here too. The property of being a space with a geodesic basis can be formulated in terms of $\mathfrak{B}_{D}(\mathbf{p}, r)$ or $\mathfrak{B}_{G}(\mathbf{p}, r)$, but it is not generally true that every $D$-ball or every $G$-ball is geodesic.

We arrive now at the standard computation of the length of a $C^{1}$ path by integration of the submetric function applied to its points and tangents.

Theorem 16 Any $C^{1}$ path $\mathbf{x} \mid[a, b]$ is smooth and smoothly parametrized, with $F(\mathbf{x}(t), \dot{\mathbf{x}}(t))=F(\mathbf{x}(t), \mathbf{x}(t))$ and

$$
D \mathbf{x}([a, b])=\int_{a}^{b} F(\mathbf{x}(t), \dot{\mathbf{x}}(t)) \mathrm{d} t .
$$

Proof. (Essentially the same as the proof of Theorem 14, because of which we only give an abridged version with a lighter notation.) By Theorem 8 ,

$$
D \mathbf{x}([a, b])=\lim _{\delta \mu \rightarrow 0} \sum D \mathbf{x}\left(t_{i}\right) \mathbf{x}\left(t_{i+1}\right)
$$


across all nets $\mu=\left\{\ldots, t_{i}, t_{i+1} \ldots\right\}$ partitioning $[a, b]$.

$$
\lim _{\delta \mu \rightarrow 0} \sum D \mathbf{x}\left(t_{i}\right) \mathbf{x}\left(t_{i+1}\right)=\lim _{\delta \mu \rightarrow 0} \sum F\left(\mathbf{x}\left(t_{i}\right), \mathbf{x}\left(t_{i+1}\right)-\mathbf{x}\left(t_{i}\right)\right) \frac{D \mathbf{x}\left(t_{i}\right) \mathbf{x}\left(t_{i+1}\right)}{F\left(\mathbf{x}\left(t_{i}\right), \mathbf{x}\left(t_{i+1}\right)-\mathbf{x}\left(t_{i}\right)\right)} .
$$

Since with $\tau-t \rightarrow 0+$ on $[a, b]$,

$$
\frac{D \mathbf{x}(t) \mathbf{x}(\tau)}{F(\mathbf{x}(t), \mathbf{x}(\tau)-\mathbf{x}(t))} \rightarrow 1
$$

(Theorem 12), we get

$$
D \mathbf{x}([a, b])=\lim _{\delta \mu \rightarrow 0} \sum F\left(\mathbf{x}\left(t_{i}\right), \mathbf{x}\left(t_{i+1}\right)-\mathbf{x}\left(t_{i}\right)\right)=\lim _{\delta \mu \rightarrow 0} \sum F\left(\mathbf{x}\left(t_{i}\right), \frac{\mathbf{x}\left(t_{i+1}\right)-\mathbf{x}\left(t_{i}\right)}{t_{i+1}-t_{i}}\right)\left(t_{i+1}-t_{i}\right) .
$$

But

$$
F\left(\mathbf{x}(t), \frac{\mathbf{x}(\tau)-\mathbf{x}(t)}{\tau-t}\right) \rightarrow F(\mathbf{x}(t), \dot{\mathbf{x}}(t))
$$

with $\tau-t \rightarrow 0+$, and $F(\mathbf{x}(t), \dot{\mathbf{x}}(t))$ is continuous. Hence

$$
D \mathbf{x}([a, b])=\lim _{\delta \mu \rightarrow 0} \sum F\left(\mathbf{x}\left(t_{i}\right), \dot{\mathbf{x}}(t)\right)\left(t_{i+1}-t_{i}\right)=\int_{a}^{b} F(\mathbf{x}(t), \dot{\mathbf{x}}(t)) \mathrm{d} t .
$$

Clearly,

$$
\lim _{s \rightarrow 0+} \frac{D \mathbf{x}(t) \mathbf{x}(t+s)}{s}=\lim _{s \rightarrow 0+} F\left(\mathbf{x}(t), \frac{\mathbf{x}(t+s)-\mathbf{x}(t)}{s}\right)=F(\mathbf{x}(t), \dot{\mathbf{x}}(t)),
$$

proving the smoothness of the $D$-parametrization and the equality

$$
F(\mathbf{x}(t), \dot{\mathbf{x}}(t))=F(\mathbf{x}(t), \stackrel{\circ}{\mathbf{x}}(t))
$$

The smoothness of $\mathbf{x} \mid[a, b]$ follows from

$$
\lim _{\tau-t \rightarrow 0+} \frac{D \mathbf{x}(t) \mathbf{x}(\tau)}{\int_{t}^{\tau} F(\mathbf{x}(t), \dot{\mathbf{x}}(t)) \mathrm{d} t}=\lim _{\tau-t \rightarrow 0+} \frac{D \mathbf{x}(t) \mathbf{x}(\tau) /(\tau-t)}{F(\mathbf{x}(t+(\tau-t) \xi), \dot{\mathbf{x}}(t+(\tau-t) \xi))}=1,
$$

by our usual argument involving $\tau, t \rightarrow c$ and the compactness of $[a, b]$.

We should mention, omitting proofs (see, e.g., Dzhafarov \& Colonius, 2001), the invariance of $D \mathbf{x}([a, b])$ for a $C^{1}$-path $\mathbf{x} \mid[a, b]$ under its diffeomorphic reparametrizations and under all diffeomorphic transformations of the space $\mathfrak{E}$.

The next theorem and the construction that follows justify one's confining one's attention to piecewise $C^{1}$ or even strictly $C^{1}$ paths only.

Theorem 17 For every rectifiable path $\mathbf{x} \mid[a, b]$ connecting $\mathbf{a}$ to $\mathbf{b}$ one can find a piecewise $C^{1}$ (in fact, piecewise linear) path from $\mathbf{a}$ to $\mathbf{b}$ which is arbitrarily close to $\mathbf{x} \mid[a, b]$ pointwise and in its $D$-length.

Proof. Due to Theorem 15, this is merely a special case of Theorem 6, with straight line segments playing the role of simple arcs.

By means of a "corner-rounding" construction mentioned in Carathéodori (1967, pp. 199-200), we can in fact replace any piecewise linear arc with a $C^{1}$-arc. Refer to Fig. 14. 


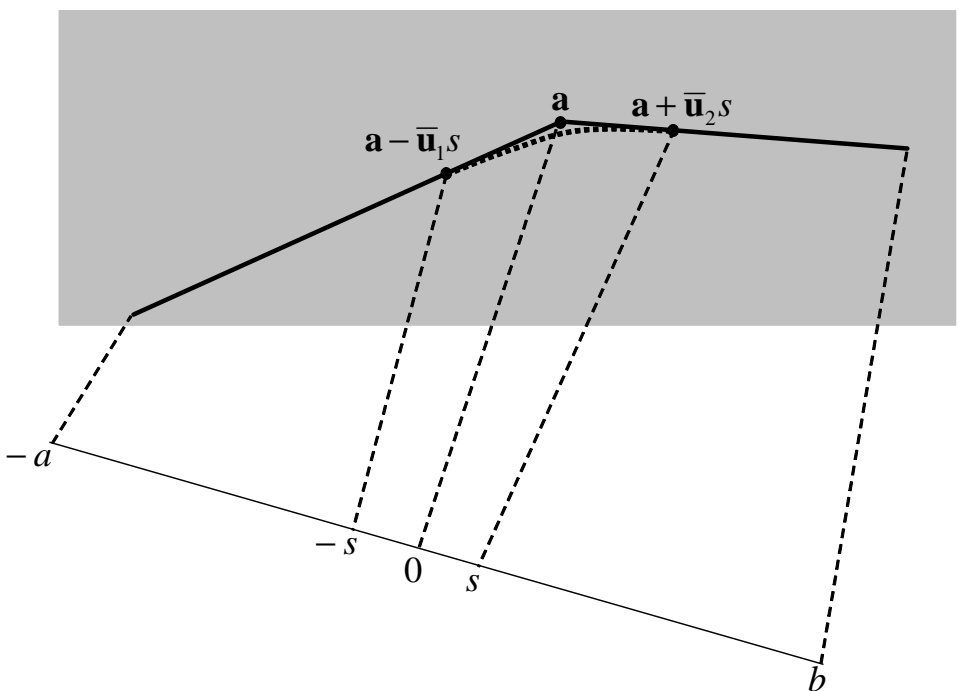

Figure 14. An illustration for the corner-rounding construction. The piecewise linear arc is shown as a mapping of the interval $[-a, b]$ into Euclidean plane (gray area). It is transformed into a $C^{1}$ arc by replacing its tip portion with the arc shown by the dotted line. The $C^{1}$ arc's $D$-length can be made arbitrarily close to that of the original, piecewise linear arc.

Let two adjacent linear pieces be parametrized as

$$
\mathbf{p}(t)= \begin{cases}\mathbf{a}+\overline{\mathbf{u}}_{1} t & \text { if } t \in[-a, 0] \\ \mathbf{a}+\overline{\mathbf{u}}_{2} t & \text { if } t \in[0, b]\end{cases}
$$

where $a, b>0$. Their combined $D$-length in a small vicinity of 0 (corresponding to their intersection point a) is

$$
D \mathbf{p}([-s, s])=\int_{-s}^{0} F\left(\mathbf{a}+\overline{\mathbf{u}}_{1} t, \overline{\mathbf{u}}_{1}\right) \mathrm{d} t+\int_{-s}^{s} F\left(\mathbf{a}+\overline{\mathbf{u}}_{2} t, \overline{\mathbf{u}}_{2}\right) \mathrm{d} t,
$$

and it obviously vanishes as we choose progressively smaller $s$. We claim that one can construct a $C^{1}$-arc

$$
\mathbf{x}(t)=\mathbf{a}+\mathbf{u}(t) t, \quad t \in[-s, s]
$$

such that

$$
\begin{aligned}
\mathbf{u}(-s) & =\overline{\mathbf{u}}_{1}, \mathbf{u}(s)=\overline{\mathbf{u}}_{2} \\
\dot{\mathbf{u}}(-s) & =\dot{\mathbf{u}}(s)=\mathbf{0}
\end{aligned}
$$

and

$$
\lim _{s \rightarrow 0+} D \mathbf{x}([-s, s])=0 .
$$

The conditions given in (44) ensure that the combined arc $\mathbf{y} \mid[-a, b]$ defined by

$$
\mathbf{y}(t)= \begin{cases}\mathbf{x}(t) & \text { if } t \in[-s, s] \\ \mathbf{p}(t) & \text { if } t \in[a,-s[\cup]-s, b]\end{cases}
$$


is $C^{1}$. Condition (45) is to ensure that the difference

$$
D \mathbf{p}([-a, b])-D \mathbf{y}([-a, b])
$$

can be made arbitrarily small by choosing $s$ sufficiently small.

It is sufficient to construct just one example of (43). Let

$$
\mathbf{u}(t)=\frac{\overline{\mathbf{u}}_{1}+\overline{\mathbf{u}}_{2}}{2}+\frac{\left(\frac{t}{s}\right)^{3}-3\left(\frac{t}{s}\right)}{4}\left(\overline{\mathbf{u}}_{1}-\overline{\mathbf{u}}_{2}\right) .
$$

One can easily verify conditions (44). One can also verify that for $-s \leq t \leq s$,

$$
\mathbf{x}(t) \in \overline{\mathfrak{B}}_{E}(\mathbf{a}, s)
$$

and

$$
|\dot{\mathbf{x}}(t)| \leq \sqrt{2}
$$

Denoting

$$
F=\max _{\substack{\mathbf{x} \in \mathfrak{B}_{E}(\mathbf{a}, s) \\ \overline{\mathbf{u}} \in \overline{\mathfrak{u}}}} F(\mathbf{x}, \overline{\mathbf{u}})
$$

we see that as $s \rightarrow 0+$,

$$
D \mathbf{x}([-s, s])=\int_{-s}^{s} F(\mathbf{x}(t), \dot{\mathbf{x}}(t)) \mathrm{d} t \leq \sqrt{2} F \cdot 2 s \rightarrow 0 .
$$

We have now rigorously developed, by means of gradually specializing the general dissimilarity-based path length theory, a version of the differential-geometric theory mentioned in Section 1.3 as the main motivation for the present work. The construction is not complete, however. For any two points $\mathbf{a}, \mathbf{b}$ we can compute, by integrating a submetric function, the $D$-length of a $C^{1}$ path connecting $\mathbf{a}$ to $\mathbf{b}$; and we know that the infimum of the $D$-lengths across all $C^{1}$ paths is the same as the infimum $A_{D}$ ab of the $D$-lengths for all paths connecting $\mathbf{a}$ to $\mathbf{b} . A_{D} \mathbf{a b}$ is an oriented distance (Dzhafarov, 2007), called the arclength metric. It is not ensured, however, that $A_{D} \mathbf{a b}$ coincides with $G \mathbf{a b}$, the oriented Fechnerian distance from $\mathbf{a}$ to $\mathbf{b}$, defined as the infimum of the $D$-lengths for all finite chains connecting $\mathbf{a}$ to $\mathbf{b}$. To ensure this we need an additional assumption.

Axiom $4(\mathfrak{E}, D)$ is a complete space with intermediate points.

In accordance with Proposition 16 we can state now that $G \mathbf{a b}$ in $(\mathfrak{E}, D)$ is an intrinsic metric in the broad Finslerian sense (see footnote 5):

$$
G \mathbf{a b}=\inf \int_{a}^{b} F(\mathbf{x}(t), \dot{\mathbf{x}}(t)) \mathrm{d} t
$$

where the infimum is taken across all $C^{1}$ paths (or piecewise $C^{1}$ paths if more convenient) connecting a to b.

Note that the completeness of $(\mathfrak{E}, D)$ cannot be derived from the completeness of $(\mathfrak{E}, E)$ because the uniformities induced by $D$ and $E$ need not coincide. 


\section{Multidimensional Fechnerian Scaling (MDFS)}

The second main postulate of UFS (the first one being the law of Regular Minimality) is that the canonical psychometric increments $\Psi^{(1)}$ and $\Psi^{(2)}$ are dissimilarity functions. An application of a DC construct formulated for an abstract dissimilarity space $(\mathfrak{S}, D)$ to UFS generally consists in ascribing the properties of $(\mathfrak{S}, D)$ in the construct to both $\left(\mathfrak{S}, \Psi^{(1)}\right)$ and $\left(\mathfrak{S}, \Psi^{(2)}\right)$. Since $\Psi^{(1)}$ and $\Psi^{(2)}$ are derived from one and the same discrimination probability function $\psi$, it often happens that postulating a property for either of $\left(\mathfrak{S}, \Psi^{(1)}\right)$ and $\left(\mathfrak{S}, \Psi^{(2)}\right)$ implies this property for the other. Thus, Proposition 5 says that $\Psi^{(1)} \mathbf{a}_{n} \mathbf{b}_{n} \rightarrow 0$ if and only if $\Psi^{(2)} \mathbf{a}_{n} \mathbf{b}_{n} \rightarrow 0$, and Proposition 17 provides another example. This is not always the case though, as one can surmise from the fact that the property of being a dissimilarity function has to be posited for both $\Psi^{(1)}$ and $\Psi^{(2)}$ rather than for just one of them. The constructs introduced in the present paper provide more examples. A path which is $\Psi^{(1)}$-smooth need not be $\Psi^{(2)}$-smooth. The property of being metric in the small for $\Psi^{(1)}$ does not imply the same for $\Psi^{(2)}$. If $\left(\mathfrak{S}, \Psi^{(1)}\right)$ is postulated to have a simple basis, the same has to be postulated for $\left(\mathfrak{S}, \Psi^{(2)}\right)$ separately, and even then the systems of simple arcs need not be the same for $\Psi^{(1)}$ and $\Psi^{(2)}$.

Focusing on the task of constructing MDFS from the abstract theory of the previous section, it is easy to see that while Axioms 1 and 4 can be posited to hold for either of $\left(\mathfrak{E}, \Psi^{(1)}\right)$ and $\left(\mathfrak{E}, \Psi^{(2)}\right)$ (and then they will hold for the other), this is not true for Axioms 2 and 3. The treatment of the axioms, however, can be made more even if we adopt an additional assumption. ${ }^{13}$

Assumption $\mathcal{E}$. For any $\mathbf{x}, \mathbf{a}_{n}, \mathbf{b}_{n} \in \mathfrak{E}\left(\mathbf{a}_{n} \neq \mathbf{b}_{n}\right)$ and any unit vector $\overline{\mathbf{u}}$, if $\mathbf{a}_{n} \leftrightarrow \mathbf{x}, \mathbf{b}_{n} \leftrightarrow \mathbf{x}$, and $\overline{\mathbf{b}_{n}-\mathbf{a}_{n}} \rightarrow \overline{\mathbf{u}}$, then

$$
\frac{\Psi^{(1)} \mathbf{a}_{n} \mathbf{b}_{n}}{\Psi^{(2)} \mathbf{b}_{n} \mathbf{a}_{n}}=\frac{\psi \mathbf{a}_{n} \mathbf{b}_{n}-\psi \mathbf{a}_{n} \mathbf{a}_{n}}{\psi \mathbf{a}_{n} \mathbf{b}_{n}-\psi \mathbf{b}_{n} \mathbf{b}_{n}}
$$

tends to a positive limit.

Theorem 18 Under Assumption $\mathcal{E}$, the space $\left(\mathfrak{E}, \Psi^{(1)}\right)$ satisfies Axioms $1-4$ if and only if so does the space $\left(\mathfrak{E}, \Psi^{(2)}\right)$.

Proof. The truth of this statement for Axioms 1 and 4 immediately follows from, respectively, Proposition 5 and Proposition 17: one does not need Assumption $\mathcal{E}$ for that. For Axiom 3 the statement is obvious. To prove the statement for Axiom 2, assume the contrary: let $\Psi^{(1)}$ be a metric in the small while $\Psi^{(2)}$ is not. Then for some sequence $\mathbf{a}_{n} \leftrightarrow \mathbf{p}, \mathbf{b}_{n} \leftrightarrow \mathbf{p}\left(\mathbf{a}_{n} \neq \mathbf{b}_{n}\right)$,

$$
\liminf _{n \rightarrow \infty} \frac{G_{2} \mathbf{a}_{n} \mathbf{b}_{n}}{\Psi^{(2)} \mathbf{a}_{n} \mathbf{b}_{n}}=1-2 \varepsilon_{0}
$$

for some positive $\varepsilon$. This follows from

$$
0<\frac{G_{2} \mathbf{a}_{n} \mathbf{b}_{n}}{\Psi^{(2)} \mathbf{a}_{n} \mathbf{b}_{n}} \leq 1 .
$$

\footnotetext{
${ }^{13}$ This assumption is equivalent to formulating Axiom 3 for both $\Psi^{(1)}$ and $\Psi^{(2)}$. We choose our approach for mathematical reasons and because it more readily lends itself to generalizations (such as Assumption $\mathcal{M}$ below).
} 
Without loss of generality, let the ratio converge to $1-2 \varepsilon_{0}$. Then for all sufficiently large $n$,

$$
\frac{G_{2} \mathbf{a}_{n} \mathbf{b}_{n}-\Psi^{(2)} \mathbf{a}_{n} \mathbf{b}_{n}}{\Psi^{(2)} \mathbf{a}_{n} \mathbf{b}_{n}}<-\varepsilon_{0} .
$$

Since $\Psi^{(1)}$ is a metric in the small, we have for the same sequence

$$
\lim _{n \rightarrow \infty} \frac{\Psi^{(1)} \mathbf{a}_{n} \mathbf{b}_{n}}{G_{1} \mathbf{a}_{n} \mathbf{b}_{n}}=1 .
$$

Then for any $\varepsilon>0$ and all sufficiently large $n$,

$$
\frac{G_{1} \mathbf{b}_{n} \mathbf{a}_{n}-\Psi^{(1)} \mathbf{b}_{n} \mathbf{a}_{n}}{\Psi^{(1)} \mathbf{b}_{n} \mathbf{a}_{n}}>-\varepsilon
$$

Rewriting the two inequalities as

$$
\begin{array}{r}
\Psi^{(2)} \mathbf{a}_{n} \mathbf{b}_{n}-G_{2} \mathbf{a}_{n} \mathbf{b}_{n}>\varepsilon_{0} \Psi^{(2)} \mathbf{a}_{n} \mathbf{b}_{n} \\
G_{1} \mathbf{b}_{n} \mathbf{a}_{n}-\Psi^{(1)} \mathbf{b}_{n} \mathbf{a}_{n}>-\varepsilon \Psi^{(1)} \mathbf{b}_{n} \mathbf{a}_{n}
\end{array}
$$

and adding them, we observe that

$$
\left(\Psi^{(2)} \mathbf{a}_{n} \mathbf{b}_{n}-G_{2} \mathbf{a}_{n} \mathbf{b}_{n}\right)+\left(G_{1} \mathbf{b}_{n} \mathbf{a}_{n}-\Psi^{(1)} \mathbf{b}_{n} \mathbf{a}_{n}\right)=0
$$

Indeed,

$$
G_{1} \mathbf{b}_{n} \mathbf{a}_{n}-G_{2} \mathbf{a}_{n} \mathbf{b}_{n}=\psi \mathbf{a}_{n} \mathbf{a}_{n}-\psi \mathbf{b}_{n} \mathbf{b}_{n}
$$

by Proposition 7 , and

$$
\Psi^{(2)} \mathbf{a}_{n} \mathbf{b}_{n}-\Psi^{(1)} \mathbf{b}_{n} \mathbf{a}_{n}=\psi \mathbf{b}_{n} \mathbf{b}_{n}-\psi \mathbf{a}_{n} \mathbf{a}_{n}
$$

by direct verification. So the two inequalities yield

$$
\varepsilon_{0} \Psi^{(2)} \mathbf{a}_{n} \mathbf{b}_{n}-\varepsilon \Psi^{(1)} \mathbf{b}_{n} \mathbf{a}_{n}<0
$$

which is equivalent to

$$
\frac{\Psi^{(1)} \mathbf{b}_{n} \mathbf{a}_{n}}{\Psi^{(2)} \mathbf{a}_{n} \mathbf{b}_{n}}>\frac{\varepsilon_{0}}{\varepsilon} .
$$

Since $\varepsilon$ can be chosen arbitrarily small, this implies

$$
\frac{\Psi^{(1)} \mathbf{b}_{n} \mathbf{a}_{n}}{\Psi^{(2)} \mathbf{a}_{n} \mathbf{b}_{n}} \rightarrow \infty .
$$

But some subsequence of $\left(\mathbf{a}_{n}, \mathbf{b}_{n}\right)$ can always be chosen so that $\overline{\mathbf{b}_{n}-\mathbf{a}_{n}} \rightarrow \overline{\mathbf{u}}$, for some $\overline{\mathbf{u}}$, and we would then arrive at a contradiction with Assumption $\mathcal{E}$. This completes the proof.

Once the complete symmetry in the treatment of $\Psi^{(1)}$ and $\Psi^{(2)}$ has been established, we know that

$$
\begin{aligned}
& F_{1}(\mathbf{a}, \mathbf{u})=\lim _{s \rightarrow 0+} \frac{\Psi^{(1)} \mathbf{a}[\mathbf{a}+\mathbf{u} s]}{s} \\
& F_{2}(\mathbf{a}, \mathbf{u})=\lim _{s \rightarrow 0+} \frac{\Psi^{(2)} \mathbf{a}[\mathbf{a}+\mathbf{u} s]}{s}
\end{aligned}
$$


are, both of them, convex functions, and the corresponding indicatrices

$$
\begin{aligned}
& \mathfrak{I}_{\mathbf{a}}^{(1)}=\left\{\mathbf{y}: F_{1}(\mathbf{a}, \mathbf{y}-\mathbf{a}) \leq 1\right\} \\
& \mathfrak{I}_{\mathbf{a}}^{(2)}=\left\{\mathbf{x}: F_{2}(\mathbf{a}, \mathbf{x}-\mathbf{a}) \leq 1\right\}
\end{aligned}
$$

are convex in the usual geometric sense. In accordance with Dzhafarov and Colonius (2001), this means that the psychometric functions $\mathbf{y} \mapsto \psi \mathbf{a y}$ and $\mathbf{x} \mapsto \psi \mathbf{x a}$ have convex cross-sections

$$
\begin{aligned}
& \mathfrak{C}_{\mathbf{x}, \psi_{0}}^{(1)}=\left\{\mathbf{y}: \psi \mathbf{a y}=\psi_{0}\right\} \\
& \mathfrak{C}_{\mathbf{x}, \psi_{0}}^{(2)}=\left\{\mathbf{x}: \psi \mathbf{x a}=\psi_{0}\right\}
\end{aligned}
$$

when $\psi_{0}$ is taken very close to the minimum level $\psi \mathbf{a a}$ (see Fig. 15).

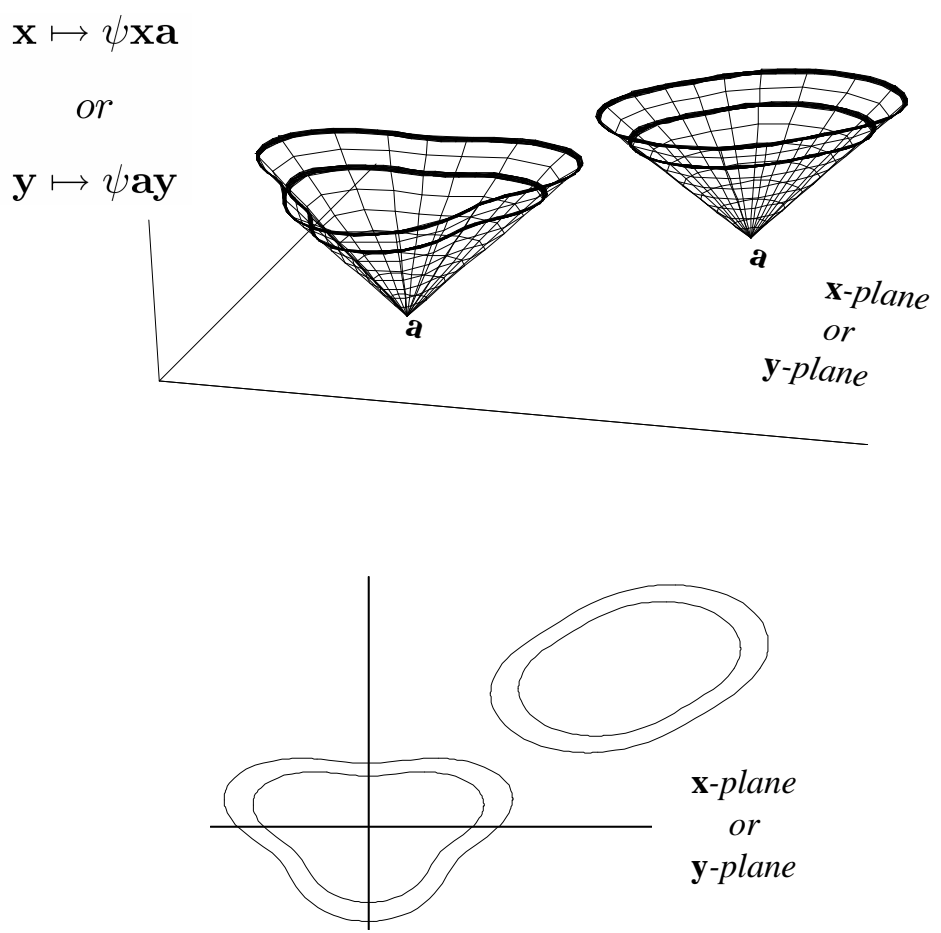

Figure 15. Two cross-sections of two hypothetical psychometric functions (of the form $\mathbf{y} \mapsto \psi \mathbf{a y}$ or $\mathbf{x} \mapsto \psi \mathbf{x a}$ ) made parallel to the canonical stimulus space (here, a plane) at very small elevations above their minima. The cross-sections are shown separately in the lower panel. According to Dzhafarov and Colonius (2001), the cross-sections approximate in shape the corresponding indicatrices, progressively better as the elevations above minima get smaller. Thus the indicatrices on the right but not on the left are convex. The reason for drawing the psychometric functions "pencil-sharp" at their minima is that the slope of the increase in the functions' values as one moves from $\mathbf{a}$ in any direction $\mathbf{u}$ equals $F_{1}(\mathbf{a}, \mathbf{u})$ (for functions $\mathbf{y} \mapsto \psi \mathbf{a y )}$ or $F_{2}(\mathbf{a}, \mathbf{u})$ (for functions $\mathbf{x} \mapsto \psi \mathbf{x a}$ ). See Dzhafarov (2002d) for a more detailed discussion.

Assumption $\mathcal{E}$ belongs to the class of "comeasurability in the small" statements for psychometric increments (see Dzhafarov, 2002d; Dzhafarov \& Colonius, 2005a). It states, essentially, that psychometric increments are of the same order of magnitude when computed between very close points. In more general settings, where direction vectors $\mathbf{u}$ are not defined, one may need weaker versions of comeasurability. Thus, 
when applying to UFS the general theory of metrics in the small, one might adopt the following generalization of Assumption $\mathcal{E}$.

Assumption $\mathcal{M}$. For any $\mathbf{p} \in \mathfrak{S}$ there is an $r>0$ such that if $\mathbf{a}_{n} \leftrightarrow \mathbf{b}_{n}\left(\mathbf{a}_{n} \neq \mathbf{b}_{n}\right)$ within $\mathfrak{B}_{D}(\mathbf{p}, r)$ then

$$
0<\liminf _{n \rightarrow \infty} \frac{\Psi^{(1)} \mathbf{a}_{n} \mathbf{b}_{n}}{\Psi^{(2)} \mathbf{b}_{n} \mathbf{a}_{n}} \leq \limsup _{n \rightarrow \infty} \frac{\Psi^{(1)} \mathbf{a}_{n} \mathbf{b}_{n}}{\Psi^{(2)} \mathbf{b}_{n} \mathbf{a}_{n}}<\infty
$$

Using the same argument as in the proof of Theorem 18 one can prove then that $\Psi^{(1)}$ is metric in the small if and only if so is $\Psi^{(2)}$.

\section{Conclusion}

We have shown that the general DC theory of path length leads to a broadly understood Finslerian theory in $n$-dimensional Euclidean spaces by means of the following assumptions, stated here informally:

1. that points very close to each other in the Euclidean sense, $|\mathbf{a}-\mathbf{b}| \rightarrow 0$, are very close in the $D$-sense too, $D \mathbf{a b} \rightarrow 0$, and vice versa;

2. that locally the dissimilarity $D$ acts like an oriented distance;

3. that neither of $|\mathbf{a}-\mathbf{b}|$ and $D \mathbf{a b}$ tends to zero infinitely faster than the other when $\mathbf{a}$ and $\mathbf{b}$ converge to a point $\mathbf{x}$ with their relative position $\mathbf{b}-\mathbf{a}$ gradually aligning with a straight line passing through $\mathbf{x}$; and

4. that for any $\mathbf{a}, \mathbf{b}$ one can find a third point $\mathbf{m}$ such that $D \mathbf{a m b}$ does not exceed $D \mathbf{a b}$.

Any path in this space can be approximated by a path with continuously turning tangents $\left(C^{1}\right)$, and the length of the latter path can be computed by means of integrating a convex submetric function which can be viewed as the magnitude of its tangent at its every point. The distance $G \mathbf{a b}$ can be found as the greatest lower bound for $D$-lengths of all $C^{1}$ paths connecting $\mathbf{a}$ to $\mathbf{b}$.

When $D$ is specified as one of the psychometric increments $\Psi^{(1)}$ and $\Psi^{(2)}$, this construction yields a version of MDFS (Dzhafarov, 2002d; Dzhafarov \& Colonius, 2001) under one additional assumption: that when points $\mathbf{a}$ and $\mathbf{b}$ converge in the manner described in the third assumption above, neither of $\Psi^{(1)} \mathbf{a b}$ and $\Psi^{(2)}$ ba tends to zero infinitely faster than the other.

It is important not to overlook that MDFS, unlike UFS, is a psycho-physical construction, in the technical sense of Dzhafarov and Colonius (2005a). This means that the truth value of its assumptions and the results of its computations depend not only on the pairwise discrimination probabilities among stimuli, but also on one's choice of the physical representation of stimuli. The logical structure of the propositions in MDSF therefore has the following form: 
There exists a canonical representation of stimuli (one in which (9) is satisfied) by an open connected region of Euclidean n-space in which the following statements (computations) are true (yield the following results): ...

If such a representation for stimuli is found, the truth of the underlying assumptions and the computation of lengths and distances remain invariant under all diffeomorphic transformations of this representation, but not under other bijective transformations. By contrast, the logical structure of the propositions in the general theory of UFS or in one of its purely psychological (in the same technical sense) specializations has the following form:

In any canonical representation of stimuli (in which (9) is satisfied), the following statements (computations) are true (yield the following results): ...

All assumptions and computational results remain therefore invariant under all possible relabelings (bijective transformations) of stimuli.

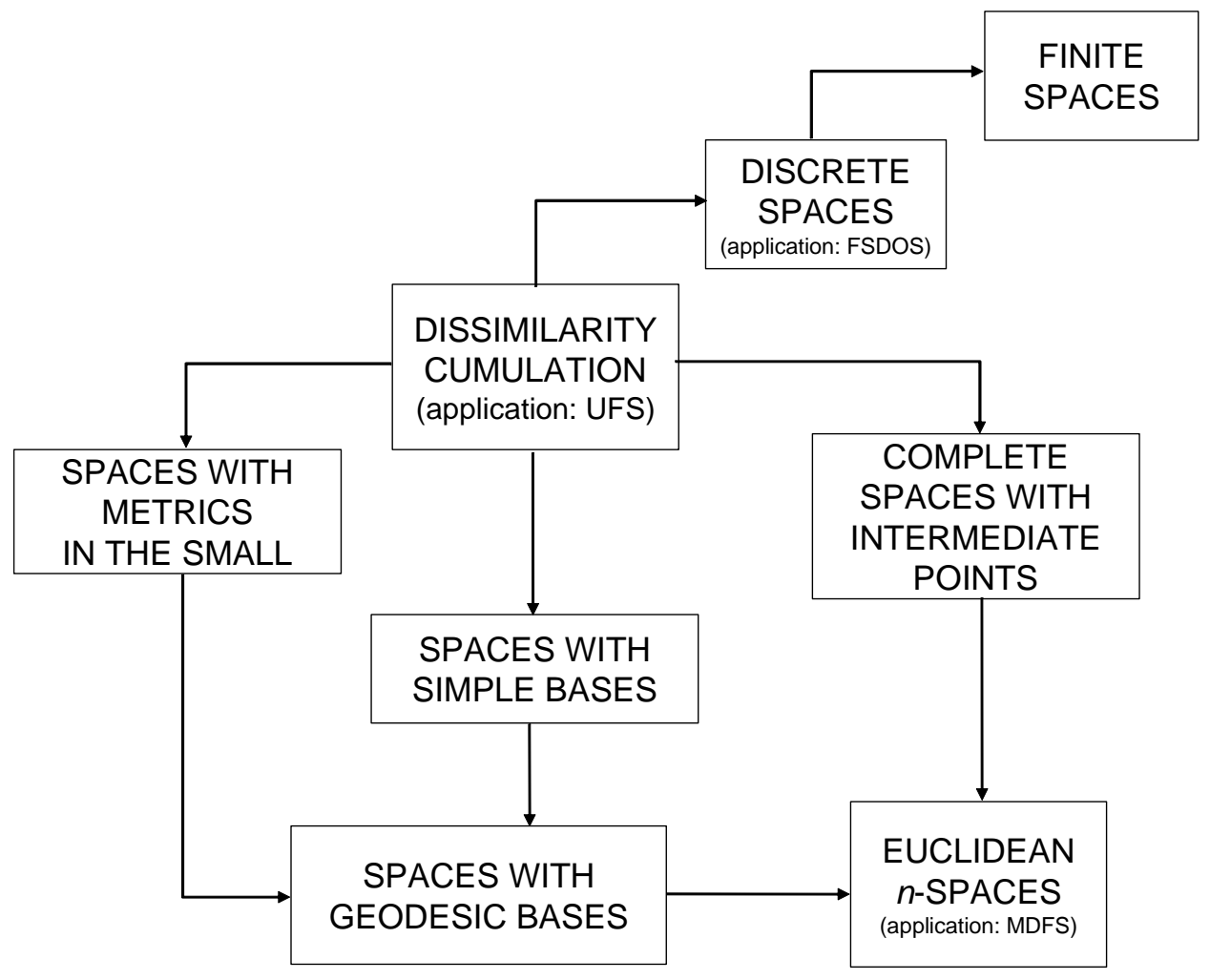

Figure 16. Various specializations of the DC theory. An arrow from A to B indicates that B is a special case of A.

The purely psychological constructions introduced in this paper on the way from the general DC theory of path length to the Finslerian one (smooth path, smoothly parametrized path, uniformly simple system of arcs, etc.) are of interest in their own right. Thus, $C^{1}$ arcs of the Euclidean space can be generalized 
into smooth arcs in arbitrary arc-connected spaces defined in terms of the dissimilarity function $D$ alone: a path is smooth if the length of its any small fragment can be approximated by the dissimilarity between the fragment's endpoints. The length of a smooth path can be computed by integrating a generalized version of the submetric function.

Figure 16 summarizes the various forms and applications of the DC theory introduced (with the exception of the discrete and finite spaces) in the series of papers of which the present one is the third. ${ }^{14}$

Acknowledgement. This research has been supported by NSF grants SES 03118010 and SES 0620446 and AFOSR grant FA9550-06-1-0288 to Purdue University. The author is grateful to James Townsend, Amir Assadi, and Janet Aisbett for corrections and helpful suggestions.

\section{References}

Blumenthal, L.M. (1953). Theory and Applications of Distance Geometry. London: Oxford University.

Blumenthal, L.M., \& Menger, K. (1970). Studies in Geometry. San Francisco, CA: W.H. Freeman.

Bruckner, A.M. (1978). Differentiation of real Functions. Berlin: Springer.

Busemann, H. (1950). The geometry of Finsler spaces. Bulletin of the American Mathematical Society, 56, $5-15$.

Busemann, H. (2005). The Geometry of Geodesics. Mineola, NY: Dover.

Carathéodori, C. (1967). Calculus of Variations and Partial Differential Equations. Part II: Calculus of Variations. San Francisco: Holden Day.

Creelman, C. D. (1967). Empirical detectability scales without the jnd. Perceptual \& Motor Skills, 24, 1079-1084.

Dzhafarov E.N. (2001). Fechnerian Psychophysics. In N.J. Smelser, P.B. Baltes (Eds.) International Encyclopedia of the Social and Behavioral Sciences, v. 8 (pp. 5437-5440). New York: Pergamon Press.

Dzhafarov, E.N. (2002a). Multidimensional Fechnerian scaling: Regular variation version. Journal of Mathematical Psychology, 46, 226-244.

Dzhafarov, E.N. (2002b). Multidimensional Fechnerian scaling: Probability-distance hypothesis. Journal of Mathematical Psychology, 46, 352-374.

\footnotetext{
${ }^{14}$ FSDOS in Fig. 16 stands for Fechnerian Scaling of Discrete Object Sets; it is also the name of software computing Fechnerian distances and geodesic chains from experimental data. The discrete and, as their special case, finite spaces have been analyzed in Dzhafarov and Colonius (2005b, 2006b-c).
} 
Dzhafarov, E.N. (2002c). Multidimensional Fechnerian scaling: Perceptual separability. Journal of Mathematical Psychology, 46, 564-582.

Dzhafarov, E.N. (2002d). Multidimensional Fechnerian scaling: Pairwise comparisons, regular minimality, and nonconstant self-similarity. Journal of Mathematical Psychology, 46, 583-608.

Dzhafarov, E.N. (2006). On the law of Regular Minimality: Reply to Ennis. Journal of Mathematical Psychology, 50, 74-93.

Dzhafarov, E.N. (2007). Dissimilarity cumulation theory in arc-connected spaces. Journal of Mathematical Psychology, in press.

Dzhafarov, E.N., \& Colonius, H. (1999). Fechnerian metrics in unidimensional and multidimensional stimulus spaces. Psychonomic Bulletin and Review, 6, 239-268.

Dzhafarov, E.N., \& Colonius, H. (2001). Multidimensional Fechnerian scaling: Basics. Journal of Mathematical Psychology, 45, 670-719.

Dzhafarov, E.N., \& Colonius, H. (2005a). Psychophysics without physics: A purely psychological theory of Fechnerian Scaling in continuous stimulus spaces. Journal of Mathematical Psychology, 49, 1-50.

Dzhafarov, E.N., \& Colonius, H. (2005b). Psychophysics without physics: Extension of Fechnerian Scaling from continuous to discrete and discrete-continuous stimulus spaces. Journal of Mathematical Psychology, 49, 125-141.

Dzhafarov, E.N., \& Colonius, H. (2006a). Regular Minimality: A fundamental law of discrimination. In H. Colonius \& E.N. Dzhafarov (Eds.), Measurement and Representation of Sensations (pp. 1-46). Mahwah, NJ: Erlbaum.

Dzhafarov, E.N., \& Colonius, H. (2006b). Generalized Fechnerian Scaling. In H. Colonius \& E.N. Dzhafarov (Eds.), Measurement and Representation of Sensations (pp. 47-88). Mahwah, NJ: Erlbaum.

Dzhafarov, E.N., \& Colonius, H. (2006c). Reconstructing distances among objects from their discriminability. Psychometrika, 71, $365-386$.

Dzhafarov, E.N., \& Colonius, H. (2007). Dissimilarity Cumulation theory and subjective metrics. Journal of Mathematical Psychology, 51, 290-304.

Falmagne, J. C. (1971). The generalized Fechner problem and discrimination. Journal of Mathematical Psychology, 8, 22-43.

Fechner, G. T. (1860). Elemente der Psychophysik [Elements of Psychophysics]. Leipzig: Breitkopf \& Härtel. Fechner, G. T. (1877). In Sachen der Psychophysik [In the matter of psychophysics]. Leipzig: Breitkopf \& Härtel. 
Fechner, G. T. (1887). Über die psychischen Massprinzipien und das Webersche Gesetz [On the principles of mental measurement and Weber's Law]. Philosophische Studien, 4, 161-230.

Helmholtz, H. von. (1891). Versuch einer erweiterten Anwendung des Fechnerschen Gesetzes im Farbensystem [An attempt at a generalized application of Fechner's Law to the color system]. Zeitschrift für die Psychologie und die Physiologie der Sinnesorgane, 2, 1-30.

Indow, T. (1994). Metrics in color spaces: Im kleinen und im grossen. In G.H. Fisher and D. Laming (Eds.) Contributions to Mathematical Psychology, Psychometrics, and Methodology (pp. 3-18). New York: Springer, 3-17.

Iverson, G.J. (2006). Analytical methods in the theory of psychophysical discrimination I: Inequalities, convexity and integration of just noticeable differences. Journal of Mathematical Psychology, 50, 271-282.

Krantz, D. (1971). Integration of just-noticeable differences. Journal of Mathematical Psychology, 8, 591599.

Pfanzagl, J. (1962). Über die stochastische Fundierung des psychophysischen Gesetzes [On stochastic foundations of the psychophysical law]. Biometrische Zeitschrift, 4, 1-14.

Robertson, A. R. (1978). CIE guidelines for coordinated research on colour-difference evaluation. Color Research E Applications, 3, 149-151.

Roweis, S.T., \& Saul, L.K. (2000). Nonlinear dimensionality reduction by locally linear embedding. Science, 290, 2323-2326.

Schrödinger, E. von. (1920). Farbenmetrik [Color metrics]. Zeitschrift für Physik, 12, 459-466.

Schrödinger, E. von (1920/1970). Outline of a theory of color measurement for daylight vision. In D.L. MacAdam (Ed.), Sources of Color Science (pp. 397-447, 481-520). Cambridge, MA: MIT Press.

Schrödinger, E. von (1926/1970). Thresholds of color differences. In D.L. MacAdam (Ed.), Sources of Color Science (pp. 183-193). Cambridge, MA: MIT Press.

Shen, Z. (2001). Lectures on Finsler Geometry. Singapore: World Scientific.

Shepard, R.N., \& Carroll, J.D. (1966). Parametric representation of nonlinear data structures. In P. R. Krishnaiah (Ed.), Multivariate Analysis (pp. 561-592). New York: Academic Press.

Tenenbaum, J.B., de Silva, V., \& Langford, J.C. (2000). A global geometric framework for nonlinear dimensionality reduction. Science, 290, 2319-2323.

Townsend, J.T., Aisbett, J., Busemeyer, J., \& Assadi, A. (2006). Methodology for dimensional independence on simple cognitive manifolds. In H. Colonius \& E.N. Dzhafarov (Eds.), Measurement and Representation of Sensations (pp. 203-241). Mahwah, NJ: Erlbaum. 
Townsend, J. T., Solomon, B., \& Spencer-Smith, J. B. (2001). The perfect Gestalt: Infinite dimensional Riemannian face spaces and other aspects of face cognition. In M. J. Wenger, \& J. T. Townsend (Eds.), Computational, Geometric and Process Issues in Facial Cognition: Progress and Challenges (pp. 39-82). Mahwah, NJ: Erlbaum.

Wyszecki, G., \& Stiles, W.S. (1982). Color Science: Concepts and Methods, Quantitative Data and Formulae. New York: Wiley

Zhang, J. (2004). Dual scaling between comparison and reference stimuli in multidimensional psychological space. Journal of Mathematical Psychology, 48: 409-424.

Zhang, J. (2006). Referential duality and representational duality in the scaling of multi-dimensional and infinite-dimensional stimulus space. In H. Colonius \& E.N. Dzhafarov (Eds.), Measurement and Representation of Sensations (pp. 131-157). Mahwah, NJ: Erlbaum. 